\title{
Herz-Schur Multipliers of Fell Bundles and the Nuclearity of the Full $C^{*}$-Algebras
}

\author{
Weijiao He \\ The Department of Mathematics, Taiyuan Normal University, Taiyuan, China
}

Email address:

whe02@qub.ac.uk

\section{To cite this article:}

Weijiao He. Herz-Schur Multipliers of Fell Bundles and the Nuclearity of the Full $C^{*}$-Algebras. International Journal of Theoretical and Applied Mathematics. Vol. 7, No. 2, 2021, pp. 17-29. doi: 10.11648/j.ijtam.20210702.11

Received: January 11, 2021; Accepted: March 18, 2021; Published: April 7, 2021

\begin{abstract}
In this paper we develop the notion of Schur multipliers and Herz-Schur multipliers to the context of Fell bundle, as a generalization of the theory of multipliers of locally compact groups and crossed products. We prove a characterization theorem of this generalized Schur multiplier in terms of the representation of Fell bundles. In order to prove this characterization theorem we define a new class of completely bounded maps; and discuss in detail of its properties. In this process, by the way, we give a new proof of Stinpring's Theorem of non-unital version. Then we investigate the transference theorem of Schur multipliers and Herz-Schur multipliers, which is a generalization of the transference theorem well-known either in the group case or crossed products. We use the notion of multipliers to define an approximation property of Fell bundles. Then we give a necessary and sufficient condition if the reduced cross-sectional algebra of a Fell bundle over a discrete groups is nuclear in terms of this generalized notion. This is a generalization of the classical theorem concerning the amenability of locally compact groups. As an application, we prove that for a Fell bundle, if its cross-sectional algebra is nuclear, then for any subgroup of the group on which the Fell bundle is defined, the cross-sectional algebra of the restricted Fell bundle on this subgroup is nuclear.
\end{abstract}

Keywords: Fell Bundles, Schur Multipliers, Herz-Schur Multipliers, Nuclearity of $C^{*}$-algebras, Approximation Property

\section{Introduction}

The notion of a Schur multiplier has its origins in the work of I. Schur in the early $20^{\text {th }}$ century, and is based on the entrywise (or Hadamard) product of matrices. More specifically, a bounded function $\varphi: \mathbb{N} \times \mathbb{N} \rightarrow \mathbb{C}$ is called a Schur multiplier if $\left(\varphi(i, j) a_{i, j}\right)$ is the matrix of a bounded linear operator on $\ell^{2}$ whenever $\left(a_{i, j}\right)$ is such. Hence to a Schur multiplier $\varphi$ : $\mathbb{N} \times \mathbb{N} \rightarrow \mathbb{C}$ we can associate an operator $S_{\varphi}$ on $\mathfrak{B}\left(\ell^{2}\right)$. Based on a concrete description of Schur multipliers which was given by A. Grothenieck in [8], Schur multipliers can be identified with $\ell^{\infty} \otimes_{e h} \ell^{\infty}$, the extended Haagerup tensor product of two copies of $\ell^{\infty}$.

Among the large number of applications of Schur multipliers is the description of the space $M A^{c b}(G)$ of completely bounded multipliers, also known as Herz-Schur multipliers of the Fourier algebra $A(G)$ of a locally compact group $\mathrm{G}$, introduced by J. de Canniere and U. Haagerup in [3]. Namely, as shown by M. Bozejko and G. Fendler [1], $M^{c b} A(G)$ can be isometrically identified with the space of all
Schur multipliers on $G \times G$ of Toeplitz type. Furthermore, in connection with Schur multiplier, Herz-Schur multiplier has very important application in the study of the nuclearity of the group $C^{*}$-algebra, i.e for a discrete group $G$, the reduced group $C^{*}$-algebra $C_{r}^{*}(G)$ is nuclear if and only if there is a net of completely positive Herz-Schur multiplier $\varphi_{i}: G \rightarrow \mathbb{C}$ such that $\varphi_{i}(x) \rightarrow 1$ for all $x \in G$ (see e.g [2]).

Recently, in [10], McKee, Todorov and Turowska generalized the notion of Schur multipliers and Herz-Schur multipliers to the $C^{*}$-algebra valued case: a class of Schur A-multipliers and a class of Herz-Schur multiplier of semidirect product bundle are identified, where $A$ is a $C^{*}$ algebra faithfully represented on a Hilbert space $H$. In this 'operator-valued' case, the starting point is a function $\phi$ defined on the direct product $X \times Y$, where $X$ and $Y$ are standard measure space, and taking values in the space $C B(A, \mathfrak{B}(H))$ of all completely bounded maps from $A$ into the $C^{*}$-algebra $\mathcal{O}(H)$ of all bounded linear operators on $H$. The associated operator $S_{\phi}$ acts from $\mathcal{O}_{c}\left(L_{2}(X), L_{2}(Y)\right) \otimes A$ into $\mathcal{O}_{c}\left(L_{2}(X), L_{2}(Y)\right) \otimes \mathcal{O}(H)$; the function $\phi$ is called 
a Schur A-multiplier if the map $S_{\phi}$ is completely bounded. Similarly, for semi-direct bundle $A \underset{\tau}{\times} G$, we start from a function $\varphi$ from $G$ into $C B(A, A)$, the associated operator $S_{\varphi}$ acts from $\mathfrak{L}_{2}(A \times G)$ (the square-integrable cross sections of $A \times G)$ to itself; the map $\varphi$ is called Herz-Schur multiplier of $A \stackrel{\tau}{\times} G$ if $S_{\varphi}$ is completely bounded with respect to the norm of $C_{r}^{*}(A \times G)$. In [9], McKee, Skalski, Todorov and Turowska use these generalized notions to generalize the classical result we mentioned in the last paragraph: for semi-direct product bundle $A \times G$ over discrete group $G, C_{r}^{*}(A \times G)$ is nuclear $C^{*}$-algebra if and only if there is a net completely positive Herz-Schur multiplier of $A \times G$ such that $\varphi_{i}(x)(a) \rightarrow a$ for all $x \in G$ and $a \in A$.

In this paper, we will generalize the notion of Herz-Schur multiplier to Fell bundle $\mathfrak{B}$ over locally compact group by borrowing the ideas of [10] and [9], then we will give a necessary and sufficient condition of that $C_{r}^{*}(\mathfrak{B})$ is nuclear $C^{*}$-algebra in terms of multipliers when $G$ is a discrete group, finally as an application we prove: If $\mathfrak{B}$ is a Fell bundle over discrete group $G$ such that $C_{r}^{*}(\mathfrak{B})$ is nuclear, then for any subgroup $H \subset G$ the $C^{*}$-algebra $C_{r}^{*}(\mathfrak{B})$ is nuclear as well.

The plan of this paper is: In the Section 2, we give some notations and conventions which we will use in this paper; in the Section 3, we define Schur multipliers of Fell bundle; in Section 4, we give a characterization of Schur multipliers of Fell bundle which is analogous to [10, Theorem 2.6], and during this process we include the non-unital version Stinpring's Theorem in a proposition which will be very important in Section 6 but with an easier proof; in Section 5 we study the generalization of Herz-Schur multipliers in the context of Fell bundles, including the generalized transference theorem between Schur multipliers and Herz-Schur multipliers (see e.g [10, Theorem 3.8]); finally in Section 6 we study the problem concerning the nuclearity of the reduced $C^{*}$-algebra by aid of the notion of the generalized Herz-Schur multipliers.

\section{Preliminary}

We refer the reader to [6, II.13] for the notion of Banach bundles, [7, VIII.2, VIII.3, VIII.16] for the notion of $C^{*}$ algebraic bundles, and [7, VIII.9] for the basic knowledge about the representation theory of $C^{*}$-algebraic bundles. As usual, we call $C^{*}$-algebraic bundles Fell bundles.

In this section we give a brief review of some basic definitions and knowledge which we will use in the rest of this paper. The definitions of notations are given in the paragraphs labeled as 'Remark'. When we use some notations we will refer to the corresponding remarks in order to remind the reader.

Remark 2.1. If $\mathfrak{D}$ is a Banach bundle over a locally compact Hausdorff space $M$ with a fixed Borel measure, we use the symbol $\mathfrak{L}(\mathfrak{D})$ to denote the space of continuous cross-sections vanishing outside some compact subset of $M$, and the symbol $\mathfrak{L}_{p}(\mathfrak{D})$ to denote the space of the $p$-integrable cross-sections of
$\mathfrak{D}$ (see [6, II.15]). If $\mathfrak{D}$ is a trivial Banach bundle with constant fiber $A$ (see $\left[6\right.$, II.13.6]), we write $\mathscr{L}(\mathscr{D})\left(\right.$ resp. $\left.\mathscr{L}_{p}(\mathscr{D})\right)$ as $\mathscr{L}(M, A)\left(\operatorname{resp} . \mathscr{L}_{p}(M, A)\right)$.

Remark 2.2. Recall that, by [6, II.15.2], if $X$ is a Hilbert space, then $\mathscr{L}_{2}(M, X)$ is a Hilbert space with the inner product

$$
\int_{M}(f(x), g(x)) d \mu x \quad\left(f, g \in \mathscr{L}_{2}(M, X)\right),
$$

where $($,$) is the inner product of \mathrm{X}$.

The following lemma is trivial, we list it here for reference:

Lemma 2.1. Let $M$ and $N$ be two locally compact Hausdorff spaces, $\phi: M \rightarrow N$ a continuous map, and $\mathfrak{D}$ a Banach bundle over $N$ with bundle space $B^{\prime}$. Let $\mathfrak{C}$ be the Banach bundle over $M$ which is the retraction of $\mathfrak{D}$ by $\phi: M \rightarrow N$, whose bundle space we denote by $Z$ (see [6, II.13.7]). If $f: M \rightarrow B^{\prime}$ is a map such that $f(m) \in B_{\phi(m)}^{\prime}$ for all $m \in M$, then the map $\widetilde{f}: M \rightarrow Z$ defined by

$$
\widetilde{f}(m)=(m, f(m)) \in Z_{m} \quad(m \in M),
$$

is a cross-section of $\mathfrak{C}$. Furthermore, $f$ is continuous if and only if $\widetilde{f}$ is continuous. We call $\widetilde{f}$ the cross-section of $\mathfrak{C}$ by canonical identification of $f$.

Remark 2.3. In the rest of this paper, we will identify $f$ and its canonical identification $\widetilde{f}$, i.e, if $f: M \rightarrow B^{\prime}$ is a (continuous) map such that $f(m) \in B_{\phi(m)}^{\prime}$, we will regard $f$ as a (continuous) cross-section of $\mathfrak{C}$.

Remark 2.4. Throughout this paper, we assume that $G$ is a fixed group which is either discrete with counting measure or is locally compact and second countable with a fixed Haar measure, and we use the symbol $\mu$ to denote either the counting measure or the Haar measure according to whether $G$ is discrete or not. Furthermore, $\mathfrak{B}=\langle B, \pi, \cdot, *\rangle$ is a fixed Fell bundle over $G$. If $G$ is not discrete, we assume that each fiber space $B_{x}$ is separable.

We refer the reader to [7, VIII.5] to see how to make $\mathscr{L}_{1}(\mathfrak{B})$ as a Banach $*$-algebra.We use the symbol $C^{*}(\mathfrak{B})$ to denote the $C^{*}$-completion (see [6, VI.10]) of $\mathscr{L}_{1}(\mathfrak{B})$.

Remark 2.5. We use the symbol $\mathfrak{B} \times \mathfrak{B}$ to denote the Banach bundle over $G \times G$ which is the retraction from $\mathfrak{B}$ by the continuous map $\phi: G \times G \rightarrow G,(x, y) \mapsto x y^{-1}$, and we denote its bundle space by $D$. For any continuous map $k: G \times G \rightarrow B$ with $k(s, t) \in B_{s t^{-1}}$, by Remark 2.3 we can regard $k$ as a continuous cross-section of $\mathfrak{B} \times \mathfrak{B}$.

Remark 2.6. In our context, if $G$ is not discrete, then by [6, Proposition II.13.21], the bundle space $B$, and so $D$ are both second countable.

Remark 2.7. Assume that $X$ is a Hilbert space. We denote the space of all bounded linear operators of $X$ by $\mathcal{O}(X)$, and the space of all compact operators by $\mathcal{O}_{c}(X)$. If $\tau$ is a representation of a Fell bundle $\mathfrak{B}$ or a $C^{*}$-algebra $A$ on $X$, sometimes we write $X$ as $X(\tau)$ in order to emphasis that the representation $\tau$ is acting on $X$.

Remark 2.8. If $\tau$ is a $*$-representation of $\mathfrak{B}$, we use the symbol $\tau_{\text {int }}$ to denote the integrated form of $\tau$ (see [7, VIII.11]). 
Remark 2.9. If $A$ is a $C^{*}$-algebra, we denote its multiplier algebra by $M(A)$. Recall that if $A$ is a concrete $C^{*}$ algebra acting on a Hilbert space $X$, then $M(A) \subset \mathcal{O}(X)$. Furthermore, if $S: A \rightarrow \mathcal{O}(Y)$ is a non-degenerate *representation of $A$ on a Hilbert space $Y$, then $S$ can be uniquely extended to $M(A)$. Therefore, we can regard $S$ as a non-degenerate ${ }^{*}$-representation of $M(A)$ on $Y$.

Definition 2.1. Recall that, by definition, the bundle space $D$ of $\mathfrak{B} \times \mathfrak{B}$ is a (topological) subspace

$$
D=\left\{(s, t ; a): s, t \in G, a \in B_{s t^{-1}}\right\}
$$

of $G \times G \times B$, and each fiber space $D_{s, t}=\{(s, t ; a): a \in$ $\left.B_{s t^{-1}}\right\}$ of $\mathfrak{B} \times \mathfrak{B}$ is isometric to $B_{s t^{-1}}(s, t \in G)$. Let us denote the subspace $\{[(s, t ; a),(t, r ; b)]: s, t, r \in G\}$ of $D \times D$ by $\mathcal{Z}$. We define the map $\diamond: \mathcal{Z} \rightarrow D$ by (recall that $a b \in B_{s r^{-1}}$ for $a \in B_{s t^{-1}}$ and $\left.b \in B_{t r^{-1}}\right)$

$$
(s, t ; a) \diamond(t, r ; b)=(s, r ; a b) \in D_{s, r},
$$

and map $*: D \rightarrow D$ by (recall that $a^{*} \in B_{t s^{-1}}$ for $a \in B_{s t^{-1}}$ )

$$
(s, t ; a)^{*}=\left(t, s ; a^{*}\right) \in D_{t, s} .
$$

Since the multiplication and involution are continuous in $B$, we conclude that $\diamond$ and $*$ are continuous.

Definition 2.2. Let $\rho: B \rightarrow \mathcal{O}(X(\rho))$ be a non-degenerate $*$-representation of $\mathfrak{B}$ such that $\left.\rho\right|_{B_{e}}$ is faithful. We define $\rho_{D}: D \rightarrow \mathcal{O}(X(\rho))$ (see Definition 2.1 for the notations) by

$$
\rho_{D}((s, t ; a)):=\rho(a) \quad((s, t ; a) \in D) .
$$

We have

$$
\begin{array}{r}
\rho_{D}((s, t ; a) \diamond(t, r ; b))=\rho_{D}((s, t ; a)) \rho_{D}((t, r ; b)) \\
((s, t ; a),(t, r ; b) \in D)
\end{array}
$$

and

$$
\begin{array}{r}
\left.\rho_{D}((s, t ; a))^{*}\right)=\rho_{D}((s, t ; a))^{*} \\
\left(s, t \in G ;(s, t ; a) \in D_{s, t}\right) .
\end{array}
$$

Furthermore, since $\rho: B \rightarrow \mathcal{O}(X(\rho))$ is strong operator continuous, for any $k \in \mathfrak{L}(\mathfrak{B} \times \mathfrak{B})$ the map $(s, t) \mapsto$ $\rho_{D}(k(s, t))$ is a strong-operator continuous map from $G \times G$ into $\mathcal{O}(X(\rho))$.

Recall that the bundle space $B$ of $\mathfrak{B}$ can be identified with a subset of $M\left(C^{*}(\mathfrak{B})\right)$ such that the topology of $B$ is stronger than the relativized topology of $B$ inherited from the strict topology of $M\left(C^{*}(\mathfrak{B})\right.$ ) (see [7] and [5]). Thus, for $a \in B$ and $b \in C^{*}(\mathfrak{B})$, we have multiplications $a b$ and $b a$ in $M\left(C^{*}(\mathfrak{B})\right)$ such that $a b \in C^{*}(\mathfrak{B})$ and $b a \in C^{*}(\mathfrak{B})$. We define $\star: D \times C^{*}(\mathfrak{B}) \rightarrow C^{*}(\mathfrak{B})$ by

$$
(s, t ; a) \star b=a b \quad\left((s, t ; a) \in D, b \in C^{*}(\mathfrak{B})\right) .
$$

For the sake of convenience, we use the same symbol $\star$ to denote the map from $C^{*}(\mathfrak{B}) \times D$ to $C^{*}(\mathfrak{B})$ defined by

$$
b \star(s, t ; a)=b a \quad\left((s, t ; a) \in D, b \in C^{*}(\mathfrak{B})\right) .
$$

By definitions we have

$$
\begin{array}{r}
\rho_{\text {int }}((s, t ; a) \star b)=\rho_{D}((s, t ; a)) \rho_{\text {int }}(b) \\
\left((s, t ; a) \in D ; b \in C^{*}(\mathfrak{B})\right),
\end{array}
$$

and similarly we have

$$
\begin{array}{r}
\rho_{\text {int }}(b \star(s, t ; a))=\rho_{\text {int }}(b) \rho_{D}((s, t ; a)) \\
\left((s, t ; a) \in D ; b \in C^{*}(\mathfrak{B})\right) .
\end{array}
$$

We state the following simple lemma without proof:

Lemma 2.2. For $k \in \mathfrak{L}(\mathfrak{B} \times \mathfrak{B}$ ) (see Remark 2.1 for the notations) and $b \in C^{*}(\mathfrak{B})$, the maps $(s, t) \mapsto k(s, t) \star b$ and $(s, t) \mapsto b \star k(s, t)$ are continuous from $G \times G$ into $C^{*}(\mathfrak{B})$.

Remark 2.10. Let $Z$ be any set, and $W \subset Z$ a subset. We use the symbol $\chi_{W}$ to denote the character function of $W$, i.e., $\chi_{W}$ is defined by

$$
\chi_{W}(x)=1 \text { if } x \in W ; \quad \chi_{W}(x)=0 \text { if } x \notin W .
$$

Remark 2.11. Our final remark is about the integration theory of Banach bundles. Let $\mathfrak{C}$ be an arbitrary Banach bundle over a locally compact Hausdorff space $M$ with Borel measure $\nu$. We use the symbol $\mathfrak{R}(\mathfrak{C})$ to denote the set of the linear span of

$$
\left\{\chi_{W} k^{\prime}: k^{\prime} \in \mathfrak{L}(\mathfrak{C}) \text { and } W \text { is compact subset of } M\right\},
$$

(see Remark 2.1 for the notations) where $\chi_{W}$ is the character function of $W$, and $\chi_{W} k^{\prime}$ is the cross-section of $\mathfrak{C}$ defined by $\chi_{W} k^{\prime}(x)=\chi_{W}(x) k^{\prime}(x)(x \in M)$. By [6, Chapter II], for any $k \in \mathfrak{L}_{p}(\mathfrak{C})$ (see Remark 2.1 for the notations) there is a sequence $\left\{k_{n}\right\}_{n \in \mathbb{N}} \subset \mathfrak{R}(\mathfrak{C})$ such that

$$
\left\|k_{n}(x)-k(x)\right\| \rightarrow 0 \quad(\text { provided } n \rightarrow \infty)
$$

and

$$
\left\|k_{n}(x)\right\| \leq\|k(x)\| \quad(n \in \mathbb{N})
$$

for almost $x \in M$. In particular, since $\left\|k_{n}(x)-k(x)\right\|^{p} \leq$ $\left(\left\|k_{n}(x)\right\|+\|k(x)\|\right)^{p}$ for almost $x \in M$ and that the function $x \mapsto\left(\left\|k_{n}(x)\right\|+\|k(x)\|\right)^{p}$ is integrable, by applying Lebesgue's Dominated Theorem, we have

$$
\int_{G}\left\|k_{n}(x)-k(x)\right\|^{p} d \nu x \rightarrow 0
$$

\section{Schur Multipliers of Fell Bundles}

In this section we define the notion of Schur multipliers of Fell bundles. We assume that $\rho$ is a $*$-representation on a Hilbert space $X$, and sometimes we will write $X$ as $X(\rho)$ (see Remark 2.7). Recall that $D$ is the bundle space of $\mathfrak{B} \times \mathfrak{B}$ (see Remark 2.5).

Proposition 3.1. Let $k \in \mathfrak{L}_{2}(\mathfrak{B} \times \mathfrak{B}$ ) (see Remark 2.1 for 
the notations). For arbitrary $\xi \in \mathfrak{L}_{2}(G, X)$ (see Remark 2.2), the map defined by

$$
P_{k, \xi}^{x}: G \rightarrow X, y \mapsto \rho_{D}(k(x, y))(\xi(y))
$$

(see Definition 2.2) is integrable for almost $x \in G$, and the map

$$
Q_{k, \xi}: G \rightarrow X, x \mapsto \int_{G} \rho_{D}(k(x, y))(\xi(y)) d y
$$

is in $\mathfrak{L}_{2}(G, X)$. Therefore, we can associate an operator $T_{k}^{\rho}$ on the Hilbert space $\mathfrak{L}_{2}(G, X)$ defined by

$$
T_{k}^{\rho}(\xi)(x)=\int_{G} \rho_{D}(k(x, y))(\xi(y)) d y \quad\left(\xi \in \mathfrak{L}_{2}(G, X)\right) .
$$

We have $\left\|T_{k}^{\rho}\right\| \leq\|k\|_{2}$.

proof. Let $k \in \mathfrak{R}(\mathfrak{B} \times \mathfrak{B})$ (see Remark 2.11 for the notations). Assume that $\xi \in \mathfrak{L}_{2}(G, X)$ having the form of $\xi=\sum_{i=1}^{n} \chi_{V_{i}} \xi_{i}^{\prime}$ (see Remark 2.10 for the notation), where $V_{i} \subset G$ are compact and $\xi_{i}^{\prime} \in X$. Then it is easy to verify that $P_{k, \xi}^{x}$ is integrable for almost $x \in G$. Furthermore, by Fubini's Theorem, $Q_{k, \xi}$ is measurable and is compactly supported. Combining with

$$
\int_{G}\left\|Q_{k, \xi}(x)\right\|^{2} d x \leq\|k\|_{2}^{2}\|\xi\|_{2}^{2},
$$

we conclude that $Q_{k, \xi}$ is in $\mathfrak{L}_{2}(G, X)$.

Now we assume that $k$ is an arbitrary element of $\mathfrak{L}_{2}(\mathfrak{B} \times \mathfrak{B})$, and $\xi$ an arbitrary element of $\mathfrak{L}_{2}(G, X)$. We prove that $P_{k, \xi}^{x}$ is integrable for almost $x \in G$. Let $\left\{\xi_{n}\right\}_{n \in \mathbb{N}}$ be a sequence of simple functions of $\mathfrak{L}_{2}(G, X)$ such that $\left\|\xi_{n}(x)-\xi(x)\right\| \rightarrow 0$ for almost $x \in G$, and $\left\|\xi_{n}-\xi\right\|_{2} \rightarrow 0$; on the other hand, let $\left\{k_{n}\right\}_{n \in \mathbb{N}}$ be a sequence of $\mathfrak{R}(\mathfrak{B} \times \mathfrak{B})$ satisfying $\left\|k_{n}(x, y)\right\| \leq$ $\|k(x, y)\|$ and $\left\|k_{n}(x, y)-k(x, y)\right\| \rightarrow 0$ in $D_{x, y}$ for almost $(x, y) \in G \times G$ (so automatically $\left\|k_{n}-k\right\|_{2} \rightarrow 0$ ). Therefore, we have a null subset $N \subset G \times G$ such that

$$
\left\|k_{n}(x, y)-k(x, y)\right\| \rightarrow 0
$$

and

$$
\left\|k_{n}(x, y)\right\| \leq\|k(x, y)\|
$$

provided $(x, y) \in(G \times G) \backslash N$. We define $N_{x}=$ $\{y \in G:(x, y) \in N\}$, and $M=\{x \in G$ : $N_{x}$ is not null subset of $\left.G\right\}$, then $M$ is a null subset of $G$, and if $x \in G \backslash M$ we have

$$
\left\|\rho_{D}\left(k_{n}(x, y)\right)\left(\xi_{n}(y)\right)-\rho_{D}(k(x, y))(\xi(y))\right\| \rightarrow 0,
$$

for almost all $y \in G$. Furthermore, there is a null set $L \subset G$ such that for all $n \in \mathbb{N}$ and $x \in G \backslash L$, the map $y \mapsto \rho_{D}\left(k_{n}(x, y)\right)\left(\xi_{n}(y)\right)$ is measurable and vanishes outside compact subsets. Combining these statements, we conclude that if $x \in G \backslash(M \cup L)$, the map $y \mapsto \rho_{D}(k(x, y))(\xi(y))$ is measurable and vanishing outside countable union of compact subsets. Moreover, there is a null set $L^{\prime} \subset G$ such that

$$
\int_{G}\|k(x, y)\|^{2} d y<\infty
$$

for $x \in G \backslash L^{\prime}$. Hence if $x \in G \backslash\left(M \cup L \cup L^{\prime}\right)$, we have

$$
\begin{aligned}
& \int_{G}\left\|\rho_{D}(k(x, y))(\xi(y))\right\| d y \leq \\
& \quad\left(\int_{G}\left\|\rho_{D}(k(x, y))\right\|^{2} d y\right)^{1 / 2} \cdot\|\xi\|_{2}<\infty .
\end{aligned}
$$

This proves that $P_{k, \xi}^{x}$ is integrable for almost $x \in G$ (i.e, for $\left.x \in G \backslash\left(M \cup L \cup L^{\prime}\right)\right)$.

Now we prove that $Q_{k, \xi}$ is in $\mathfrak{L}_{2}(G, X)$. Our first step is to verify that $Q_{k, \xi}$ is measurable. For $x \in G \backslash\left(M \cup L \cup L^{\prime}\right)$ we have

$$
\begin{aligned}
& \| \int_{G}\left(\rho_{D}(k(x, y))(\xi(y))-\rho_{D}\left(k_{n}(x, y)\right)\left(\xi_{n}(y)\right) d y \|\right. \\
& \leq\left(\int_{G}\left\|k_{n}(x, y)-k(x, y)\right\|^{2} d y\right)^{1 / 2} \cdot\|\xi\|_{2} \\
& +\left(\int_{G}\left\|k_{n}(x, y)\right\|^{2} d y\right)^{1 / 2} \cdot\left\|\xi-\xi_{n}\right\|_{2} .
\end{aligned}
$$

By our definitions of the null subsets $L, M, L^{\prime} \subset G$, if $x \in G \backslash\left(M \cup L \cup L^{\prime}\right)$, the sequence $\left\{\int_{G}\left\|k_{n}(x, y)\right\|^{2} d y\right\}_{n \in \mathbb{N}}$ is bounded, so we have

$$
\| \int_{G}\left(\rho_{D}(k(x, y))(\xi(y))-\rho_{D}\left(k_{n}(x, y)\right)\left(\xi_{n}(y)\right) d y \| \rightarrow 0\right.
$$

for $x \in G \backslash\left(M \cup L \cup L^{\prime}\right)$. This proves that $Q_{k, \xi}$ is measurable, and it is clear that $Q_{k, \xi}$ is vanishing outside a countable union of compact subsets of $G$. Furthermore, by the same argument which derived (10), we have

$$
\int_{G}\left\|Q_{k, \xi}(x)\right\|^{2} d x \leq\|k\|_{2}^{2}\|\xi\|_{2}^{2} .
$$

We conclude that $Q_{k, \xi}$ is in $\mathfrak{L}_{2}(G, X)$.

The other parts of this proposition is easy consequence of our previous discussion.

Remark 3.1. We use the symbol $\mathfrak{E}(\rho, \mathfrak{B})$ to denote the normclosure of the set $\left\{T_{k}^{\rho}: k \in \mathfrak{L}_{2}(\mathfrak{B} \times \mathfrak{B})\right\}$ in $\mathcal{O}\left(\mathfrak{L}_{2}(G, X)\right)$, (see (9), Remark 2.7 and Remark 2.2 for the notations).

Lemma 3.1. If $k_{1}, k_{2} \in \mathfrak{L}(\mathfrak{B} \times \mathfrak{B})$, the map defined by

$$
G \rightarrow D_{x, y}, z \mapsto k_{1}(x, z) \diamond k_{2}(z, y)
$$

(see Definition 2.1 for the notations) is continuous and compactly supported for all $(x, y) \in G \times G$, and the map

$$
J: G \times G \rightarrow D_{x, y},(x, y) \mapsto \int_{G} k_{1}(x, z) \diamond k_{2}(z, y) d z
$$

is in $\mathfrak{L}(\mathfrak{B} \times \mathfrak{B})$.

proof. Since $k_{1}$ and $k_{2}$ are continuous cross-sections, the 
map

$$
p:(x, z, y) \mapsto\left(x, y ; c_{k_{1}}(x, z) c_{k_{2}}(z, y)\right)
$$

is a continuous from $G \times G \times G$ into $D$, where $c_{k_{i}}: G \times G \rightarrow B$ is the map satisfying $k_{i}(x, y)=\left(x, y ; c_{k_{i}}(x, y)\right)(i=1,2)$ (see Remark2.1), and, since $k_{i}(i=1,2)$ is compactly supported, so is $p$. Furthermore, by the continuity of $p$ and the equality

$$
k_{1}(x, z) \diamond k_{2}(z, y)=p(x, y, z)
$$

we conclude that the map $z \mapsto k_{1}(x, z) \diamond k_{2}(z, y)$ is continuous. In order to complete our proof, since

$$
\begin{aligned}
\int_{G} p(x, z, y) d z & =\int_{G}\left(x, y ; c_{k_{1}}(x, z) c_{k_{2}}(z, y)\right) d z \\
& =\int_{G} k_{1}(x, z) \diamond k_{2}(z, y) d z
\end{aligned}
$$

it is sufficient to prove that

$$
(x, y) \mapsto \int_{G} p(x, z, y) d z
$$

is continuous from $G \times G$ into $D$. But, by [6, II.15.19], (13) is continuous cross-section of $\mathfrak{B} \times \mathfrak{B}$. Our proof is complete.

Definition 3.1. For $k_{1}, k_{2} \in \mathfrak{L}(\mathfrak{B} \times \mathfrak{B})$, we use the symbol $k_{1} \star k_{2}$ to denote the cross-section of $\mathfrak{B} \times \mathfrak{B}$ defined by

$$
(x, y) \mapsto \int_{G} k_{1}(x, z) \diamond k_{2}(z, y) d z\left(\in B_{x y^{-1}}\right),
$$

(recall that by Lemma $3.1, k_{1} \star k_{2} \in \mathfrak{L}(\mathfrak{B} \times \mathfrak{B})$ ), and the symbol $k_{1}^{*}$ to denote the cross-section defined by

$$
(x, y) \mapsto k_{1}(y, x)^{*}\left(\in B_{x y^{-1}}\right) .
$$

The following lemma can be verified by routine computation, we omit its proof:

Lemma 3.2. For any $k_{1}, k_{2} \in \mathfrak{L}(\mathfrak{B} \times \mathfrak{B})$, we have (see (9) and Definition 3.1 for the notations)

$$
\begin{aligned}
& T_{k_{1} \star k_{2}}^{\rho}=T_{k_{1}}^{\rho} T_{k_{2}}^{\rho}, \\
& \left(T_{k_{1}}\right)^{*}=T_{k_{1}^{*}}^{\rho} .
\end{aligned}
$$

Therefore, $\mathfrak{E}(\rho, \mathfrak{B})$ (see Remark 3.1 for the notations) is a $C^{*}$-algebra.

Remark 3.2. We denote the set of all simple functions $l \in$ $\mathfrak{L}_{2}\left(G \times G, C^{*}(\mathfrak{B})\right)$ (see Remark 2.1 for the notations) having the form of

$$
l=\sum_{i=1}^{n} a_{i} \chi_{E_{i} \times F_{i}},\left(a_{i} \in C^{*}(\mathfrak{B}), E_{i}, F_{i} \subset G \text { are compact }\right)
$$

by $\mathfrak{K}(\mathfrak{B})$. Since, by [6, II.9.2] and [6, II.9.4], the linear span of

$$
\left\{\chi_{E \times F}: E \text { and } F \text { are compact }\right\}
$$

is dense in $\mathfrak{L}_{2}(G \times G)$, it is clear that $\mathfrak{K}(\mathfrak{B})$ is dense in $\mathfrak{L}_{2}\left(G \times G, C^{*}(\mathfrak{B})\right)$.
Since we assumed that $\rho$ is a non-degenerate *representation, the concrete $C^{*}$-algebra $\mathcal{O}_{c}\left(\mathfrak{L}_{2}(G)\right) \otimes$ $\rho_{\text {int }}\left(C^{*}(\mathfrak{B})\right)$ acts on $\mathfrak{L}_{2}(G, X)$ non-degenerately, hence we have $M\left(\mathcal{O}_{c}\left(\mathfrak{L}_{2}(G)\right) \otimes \rho_{\text {int }}\left(C^{*}(\mathfrak{B})\right)\right) \subset \mathcal{O}\left(\mathfrak{L}_{2}(G, X)\right)$ (see Remark 2.9). In Lemma 3.3 below, we will prove that $\mathfrak{E}(\rho, \mathfrak{B}) \subset M\left(\mathcal{O}_{c}\left(\mathfrak{L}_{2}(G)\right) \otimes \rho_{\text {int }}\left(C^{*}(\mathfrak{B})\right)\right)$.

Remark 3.3. Let us recall from [10] that, for any $k^{\prime} \in$ $\mathfrak{L}_{2}\left(G \times G, C^{*}(\mathfrak{B})\right)$, the operator $T_{k^{\prime}}^{\rho_{\text {int }}}$ on $\mathfrak{L}_{2}(G, X)$ defined by

$$
T_{k^{\prime}}^{\rho_{\text {int }}}(\xi)(x)=\int \rho_{\text {int }}\left(k^{\prime}(x, y)\right)(\xi(y)) d y \quad\left(\xi \in \mathfrak{L}_{2}(G, X)\right)
$$

is in $\mathcal{O}_{c}\left(\mathfrak{L}_{2}(G)\right) \otimes \rho_{\text {int }}\left(C^{*}(\mathfrak{B})\right)$.

Lemma 3.3. Let $k \in \mathfrak{L}(\mathfrak{B} \times \mathfrak{B})$ and $l \in \mathfrak{K}(\mathfrak{B})$. The following maps from $G$ into $C^{*}(\mathfrak{B})$

$$
\begin{aligned}
& z \mapsto k(x, z) \star l(z, y), \\
& z \mapsto l(z, y) \star k(x, z)
\end{aligned}
$$

are in $\mathfrak{L}_{1}\left(G, C^{*}(\mathfrak{B})\right)$ for almost $(x, y) \in G \times G$, and the maps $k \star l$ and $l \star k$ defined by

$$
\begin{array}{ll}
k \star l(x, y) & =\int_{G} k(x, z) \star l(z, y) d z \quad(x, y \in G), \\
l \star k(x, y) & =\int_{G} l(x, z) \star k(z, y) d z \quad(x, y \in G)
\end{array}
$$

(see (7) and (8) for the notations) are in $\mathfrak{L}_{2}\left(G \times G, C^{*}(\mathfrak{B})\right.$ ). Furthermore we have (see Proposition 3.1 and Remark 3.3 for the notations)

$$
T_{k}^{\rho} T_{l}^{\rho_{\mathrm{int}}}=T_{k \star l}^{\rho_{\mathrm{int}}} ; T_{l}^{\rho_{\mathrm{int}}} T_{k}^{\rho}=T_{l \star k}^{\rho_{\mathrm{int}}} .
$$

In particular, since $\left\{T_{l}^{\rho_{\text {int }}}: l \in \mathfrak{K}(\mathfrak{B})\right\}$ is dense in $\mathcal{O}_{c}\left(\mathfrak{L}_{2}(G)\right) \otimes \rho_{\text {int }}\left(C^{*}(\mathfrak{B})\right)$, we have $\mathfrak{E}(\rho, \mathfrak{B}) \subset$ $M\left(\mathcal{O}_{c}\left(\mathfrak{L}_{2}(G)\right) \otimes \rho_{\text {int }}\left(C^{*}(\mathfrak{B})\right)\right)$.

proof. Let $l$ be a function with the form of (14). Suppose $\operatorname{supp}(k) \subset E \times F$, where $E$ and $F$ are compact subsets of $G$. Our first task is to prove that the map $z \mapsto k(x, z) \star l(z, y)$ is in $\mathfrak{L}_{1}\left(G, C^{*}(\mathfrak{B})\right)$ for almost $(x, y) \in G \times G$, the second is to prove that $(x, y) \mapsto \int_{G} k(x, z) \star l(z, y) d z$ is in $\mathfrak{L}_{2}(G \times$ $\left.G, C^{*}(\mathfrak{B})\right)$, and finally we verify $T_{k}^{\rho} T_{l}^{\rho_{\text {int }}}=T_{k \star l}^{\rho_{\text {int }}}$.

Let define $q: G \times G \times G \rightarrow C^{*}(\mathfrak{B})$ by

$$
\begin{aligned}
q(x, z, y) & =k(x, z) \star l(z, y) \\
& =\sum_{i=1}^{n}\left(k(x, z) \star a_{i}\right) \chi_{E_{i} \times F_{i}}(z, y) .
\end{aligned}
$$

Then $q$ is bounded, measurable and compactly supported by $E \times\left(\bigcup_{i=1}^{n} E_{i}\right) \times\left(\bigcup_{i=1}^{n} F_{i}\right)$. In particular, $q \in \mathfrak{L}_{1}(G \times G \times$ $\left.G, C^{*}(\mathfrak{B})\right)$. By Fubini's Theorem, the map $z \mapsto k(x, z) \star$ $l(z, y)$ is in $\mathfrak{L}_{1}\left(G, C^{*}(\mathfrak{B})\right)$ for almost $(x, y) \in G \times G$.

For any fixed $x$ and $y$, we define $p_{x, y}: G \rightarrow C^{*}(\mathfrak{B})$ by $p_{x, y}(z)=q(x, z, y)(=k(x, z) \star l(z, y))$. Then, by the fact that we proved $q \in \mathfrak{L}_{1}\left(G \times G \times G, C^{*}(\mathfrak{B})\right)$ and Fubini's Theorem, the map $k \star l:(x, y) \mapsto \int_{G} p_{x, y}(z) d z$ is measurable. On the 
other hand, since $q$ is compactly supported by $E \times\left(\bigcup_{i=1}^{n} E_{i}\right) \times$ $\left(\bigcup_{i=1}^{n} F_{i}\right)$ and bounded, we conclude that $k \star l:(x, y) \mapsto$ $\int_{G} p_{x, y}(z) d z=\int_{G} q(x, z, y) d z$ is bounded, measurable and compactly supported by $E \times \bigcup_{i=1}^{n} F_{i}$. Therefore, we conclude that $k \star l \in \mathfrak{L}_{2}\left(G \times G, C^{*}(\mathfrak{B})\right)$.

By our previous discussion, for almost $(x, y) \in G \times G$ we have $C^{*}(\mathfrak{B})$-valued integration $\int_{G} k(x, z) \star l(z, y) d z$. Hence, by the norm contunuity of $\rho_{\text {int }}: C^{*}(\mathfrak{B}) \rightarrow \mathcal{O}(X)$, we have

$\rho_{\mathrm{int}}\left(\int_{G} k(x, z) \star l(z, y) d z\right)=\int_{G} \rho_{\mathrm{int}}(k(x, z) \star l(z, y)) d z$,

for almost $(x, y) \in G \times G$. Therefore, for any fixed $\xi \in$ $\mathfrak{L}_{2}(G, X)$, we have

$$
\begin{aligned}
T_{k \star l}^{\rho_{\text {int }}} \xi(x) & =\int_{G} \rho_{\text {int }}\left(\int_{G} k(x, z) \star l(z, y) d z\right) \xi(y) d y \\
& =T_{k}^{\rho^{2}} T_{l}^{\rho_{\text {int }}} \xi(x),
\end{aligned}
$$

for almost $x \in G$. This proves that $T_{k \star l}^{\rho_{\text {int }}}=T_{k}^{\rho} T_{l}^{\rho_{\text {int }}}$. $T_{l}^{\rho_{\text {int }}} T_{k}^{\rho}=T_{l \star k}^{\rho_{\text {int }}}$ can be proved by the same argument.

Lemma 3.4. Let $r: B \rightarrow \mathcal{O}(Y)$ be a non-degenerate *representation of $\mathfrak{B}$ on a Hilbert space $Y$ which is weakly contained in $\rho$. Then the following map (see Remark 3.3 for the notations)

$$
\Xi_{\rho, r}: T_{k^{\prime}}^{\rho_{\text {int }}} \mapsto T_{k^{\prime}}^{r_{\text {int }}} \quad\left(k^{\prime} \in \mathfrak{L}_{2}\left(G \times G, C^{*}(\mathfrak{B})\right)\right)
$$

can be extended to a $*$-homomorphism from $\mathcal{O}_{c}\left(\mathfrak{L}_{2}(G)\right) \otimes$ $\rho_{\text {int }}(\mathfrak{B})$ onto $\mathcal{O}_{c}\left(\mathfrak{L}_{2}(G)\right) \otimes r_{\text {int }}(\mathfrak{B})$. Therefore, we can regard $\Xi_{\rho, r}$ as a *-homomorphism from $M\left(\mathcal{O}_{c}\left(\mathfrak{L}_{2}(G)\right) \otimes \rho_{\text {int }}(\mathfrak{B})\right)$ onto $M\left(\mathcal{O}_{c}\left(\mathfrak{L}_{2}(G)\right) \otimes r_{\text {int }}(\mathfrak{B})\right)$ (see Remark 2.9) satisfying (see (9) for the notation)

$$
\Xi_{\rho, r}\left(T_{k}^{\rho}\right)=T_{k}^{r} \quad\left(k \in \mathfrak{L}_{2}(\mathfrak{B} \times \mathfrak{B})\right)
$$

and

$$
\begin{gathered}
\Xi_{\rho, r}\left(T_{k}^{\rho}\right) \Xi_{\rho, r}\left(T_{l}^{\rho_{\mathrm{int}}}\right)=T_{k}^{r} T_{l}^{r_{\mathrm{int}}} \\
\quad(k \in \mathfrak{L}(\mathfrak{B} \times \mathfrak{B}) ; l \in \mathfrak{K}(\mathfrak{B})) .
\end{gathered}
$$

proof. Since $r$ is weakly contained in $\rho$, the map $S$ : $\rho_{\text {int }}\left(C^{*}(\mathfrak{B})\right) \rightarrow r_{\text {int }}\left(C^{*}(\mathfrak{B})\right)$ defined by $S\left(\rho_{\text {int }}(a)\right)=r_{\text {int }}(a)$ $\left(a \in C^{*}(\mathfrak{B})\right)$ is $\mathrm{a}^{*}$-homomorphism. Then the map $S \otimes$ $Q$, where $Q$ is the identity representation of $\mathcal{O}_{c}\left(\mathfrak{L}_{2}(G)\right)$ on $\mathfrak{L}_{2}(G)$, is a *-homomorpphism from $\mathcal{O}_{c}\left(\mathfrak{L}_{2}(G)\right) \otimes \rho_{\text {int }}(\mathfrak{B})$ onto $\mathcal{O}_{c}\left(\mathfrak{L}_{2}(G)\right) \otimes r_{\text {int }}(\mathfrak{B})$, which is an extension of $\Xi_{\rho, r}$.

For each $k \in \mathfrak{L}(\mathfrak{B} \times \mathfrak{B}$ ) and $l \in \mathfrak{K}(\mathfrak{B})$ (see Remark 3.2 for the notations), by Lemma 3.3, we have

$$
\begin{aligned}
& \left.\left((S \otimes Q)\left(T_{k}^{\rho}\right)\right)(S \otimes Q)\left(T_{l}^{\rho_{\mathrm{int}}}\right)\right) \\
= & T_{k}^{r} \circ\left((S \otimes Q)\left(T_{l}^{\rho_{\mathrm{int}}}\right)\right) .
\end{aligned}
$$

Since $\left\{T_{l}^{\rho_{\text {int }}}: l \in \mathfrak{K}(\mathfrak{B})\right\}$ is dense in $\mathcal{O}_{c}\left(\mathfrak{L}_{2}(G)\right) \otimes$ $r_{\text {int }}\left(C^{*}(\mathfrak{B})\right)$ and $S \otimes I_{\mathfrak{L}_{2}(G)}$ is non-degenerate, (18) implies that

$$
\Xi_{\rho, r}\left(T_{k}^{\rho}\right)=(S \otimes Q)\left(T_{k}^{\rho}\right)=T_{k}^{r} \quad(k \in \mathfrak{L}(\mathfrak{B} \times \mathfrak{B})) .
$$

Now (16) is proved for $k \in \mathfrak{L}(\mathfrak{B} \times \mathfrak{B})$. But since $\mathfrak{L}(\mathfrak{B})$ is dense in $\mathfrak{L}_{2}(\mathfrak{B}),(16)$ holds for $k \in \mathfrak{L}_{2}(\mathfrak{B} \times \mathfrak{B})$.

(17) is a direct consequence of Lemma 3.3.

The proof is complete.

Proposition 3.2. Let $k \in \mathfrak{L}_{2}(\mathfrak{B} \times \mathfrak{B})$. Then $T_{k}^{\rho}=0$ if and only if $k(x, y)=0$ for almost all $(x, y) \in G \times G$.

proof. The 'if' part is trivial, we prove the 'only if' part. Let $R: \rho_{\text {int }}\left(C^{*}(\mathfrak{B})\right) \rightarrow \mathcal{O}(Y)$ be a non-degenerate faithful $*$ representation of $\rho_{\text {int }}\left(C^{*}(\mathfrak{B})\right)$ on a separable Hilbert space Y, and $r: B \rightarrow \mathcal{O}(Y)$ the ${ }^{*}$-representation of $\mathfrak{B}$ on $Y$ such that $R \circ \rho_{\text {int }}$ is the integrated form of $r$. Since $\rho$ and $r$ are weakly equivalent, the map $\Xi_{\rho, r}$, defined in Lemma 3.4, is faithful *homomorhpism, hence $T_{k}^{\rho}=0$ implies that $T_{k}^{r}=0$. Since $\rho_{\text {int }}\left(C^{*}(\mathfrak{B})\right)$ is a separable $C^{*}$-algebra, by [10], $T_{k}^{r}=0$ if and only if $k(x, y)=0$ for almost all $(x, y) \in G \times G$. Our proof is complete.

Definition 3.2. Let $\mathfrak{D}$ be a Banach bundle over locally compact space $\mathrm{M}$ with bundle space $D^{\prime}$. We call a continuous map $\Phi: D^{\prime} \rightarrow D^{\prime}$ multiplier of $\mathfrak{D}$ if $\Phi$ satisfies:

(i) $\Phi\left(D_{x}^{\prime}\right) \subset D_{x}^{\prime}$ for all $x \in M$;

(ii) $\Phi \mid D_{x}^{\prime}$ is bounded linear map such that $\sup _{x \in M}\left\|\Phi \mid D_{x}^{\prime}\right\|<\infty$.

Let $\Phi: D \rightarrow D$ be a multiplier of $\mathfrak{B} \times \mathfrak{B}$. We denote $\sup _{(x, y) \in G \times G}\left\|\Phi \mid D_{x, y}\right\|$ by $M$. For any $k \in \mathfrak{L}_{2}(\mathfrak{B} \times \mathfrak{B})$, we define $\Phi \cdot k(x, y)=\Phi(k(x, y))(x, y \in G)$, then $\Phi \cdot k \in$ $\mathfrak{L}_{2}(\mathfrak{B} \times \mathfrak{B})$.

Definition 3.3. Let $\Phi: D \rightarrow D$ be a multiplier of $\mathfrak{B} \times \mathfrak{B}$. If the map $S_{\rho, \Phi}:\left\{T_{k}^{\rho}: k \in \mathfrak{L}_{2}(\mathfrak{B})\right\} \rightarrow \mathcal{O}\left(\mathfrak{L}_{2}(G, X(\rho))\right)$ defined by

$$
S_{\rho, \Phi}\left(T_{k}^{\rho}\right)=T_{\Phi \cdot k}^{\rho},
$$

is completely bounded linear map, then we say that $\Phi$ is a $\operatorname{Schur}(\mathfrak{B}, \rho)$-multiplier. Fuarthermore, if $S_{\rho}, \Phi$ is completely positive, we say $\Phi$ is completely positive $\operatorname{Schur}(\mathfrak{B}, \rho)$ multiplier.

If $\Phi$ is a $\operatorname{Schur}(\mathfrak{B}, \rho)$-multiplier, we define

$$
\|\Phi\|_{\mathfrak{S}, \rho}:=\left\|S_{\rho, \Phi}\right\|_{\mathrm{cb}} .
$$

Therefore, if $\Phi$ is a $\operatorname{Schur}(\mathfrak{B}, \rho)$-multiplier, $S_{\rho, \Phi}$ can be extended to a completely bounded map from $\mathfrak{E}(\rho, \mathfrak{B})$ (see Remark 3.1 for the notations) into $\mathcal{O}\left(\mathfrak{L}_{2}(G, X(\rho))\right)$. Thus we will always consider that $S_{\rho, \Phi}$ is a completely bounded map defined on $\mathfrak{E}(\rho, \mathfrak{B})$.

The following proposition is useful in our further study:

Proposition 3.3. If $r: B \rightarrow \mathcal{O}(Y)$ is a non-degenerate $*$ representation of $\mathfrak{B}$ on a Hilbert space $Y$ which is weakly equivalent to $\rho$, then $\Phi$ is a $\operatorname{Schur}(\mathfrak{B}, r)$-multiplier if and only if it is a $(\mathfrak{B}, \rho)$-multiplier.

proof. Since $r$ is weakly to $\rho$, by Lemma 3.4, the restriction of $\Xi_{\rho, r}$ on $\mathfrak{E}(\rho, \mathfrak{B})$, i.e

$$
\left.\Xi_{\rho, r}\right|_{\mathfrak{E}(\rho, \mathfrak{B})}: \mathfrak{E}(\rho, \mathfrak{B}) \rightarrow \mathfrak{E}(r, \mathfrak{B}), T_{k}^{\rho} \mapsto T_{k}^{r}(k \in \mathfrak{L}(\mathfrak{B} \times \mathfrak{B})),
$$

is $*$-isomomorphism from $\mathfrak{E}(\rho, \mathfrak{B})$ onto $\mathfrak{E}(r, \mathfrak{B})$ with inverse $\left.\Xi_{r, \rho}\right|_{\mathfrak{E}(r, \mathfrak{B})}$.

Suppose that $\Phi$ is a $\operatorname{Schur}(\mathfrak{B}, \rho)$-multiplier. Then, since $S_{\rho, \Phi}: \mathfrak{E}(\rho, \mathfrak{B}) \rightarrow \mathfrak{E}(\rho, \mathfrak{B})$ is completely bounded, $\Xi_{\rho, r} \circ$ 
$S_{\rho, \Phi} \circ \Xi_{\rho, r}^{-1}: \mathfrak{E}(r, \mathfrak{B}) \rightarrow \mathfrak{E}(r, \mathfrak{B})$ is completely bounded satisfying $\Xi_{\rho, r} \circ S_{\rho, \Phi} \circ \Xi_{\rho, r}^{-1}=S_{r, \Phi}$. Hence $\Phi$ is s Schur $(\mathfrak{B}, r)$-multiplier. By the same argument, we can prove that if $\Phi$ is a $\operatorname{Schur}(\mathfrak{B}, r)$-multiplier, then $\Phi$ is a $\operatorname{Schur}(\mathfrak{B}, \rho)$ multiplier.

\section{Characterization of Schur $(\mathfrak{B}, \rho)$-multipliers}

Our main result of this section is the characterization of Schur $(\mathfrak{B}, \rho)$ - multipliers (See Definition 3.3). In order to achieve this goal, we define a specific class of completely bounded maps in Definition 4.2.

In the rest of this section, let $A$ be a fixed $C^{*}$-algebra, $M(A)$ the multiplier algebra of $A$ (see Remark 2.9), and $X$ a fixed Hilbert space. Recall that $\mathcal{O}(X)$ is the space of all bounded linear operators of $X$.

Definition 4.1. Let $f: A \rightarrow \mathcal{O}(X)$ be a completely bounded map. $(W, V, R, Y)$, consisting of a representation $R$ of $A$ on a Hilbert space $Y$ and bounded operators $W, V: X \rightarrow Y$ satisfying:

$$
f(a)=W^{*} R_{a} V \quad(a \in A),
$$

is said to be a representation of $f$. If $R$ is non-degenerate, we say that $(W, V, R, Y)$ is non-degenerate.

If $W=V$, we write $(V, V, R, Y)$ as $(V, R, Y)$ or $(V, R)$.

Definition 4.2. By a completely bounded map from $M(A)$ into $\mathcal{O}(X)$ with Property $\left(S_{A}\right)$ or briefly a $\left(S_{A}\right)$-map on M(A), we shall mean a completely bounded linear map $f: M(A) \rightarrow$ $\mathcal{O}(X)$ which is continuous with respect to the strict topology of $M(A)$ and strong* topology of $\mathcal{O}(X)$ on the norm bounded subsets of $M(A)$.

Let $C \subset M(A)$ be a $C^{*}$-subalgebra. By a completely bounded $\left(S_{C, A}\right)$-extendable map from $C$ into $\mathcal{O}(X)$ or briefly a $\left(S_{C, A}\right)$-extendable map on $C$, we shall mean a completely bounded map $g: C \rightarrow \mathcal{O}(X)$ which has an extension on $M(A)$ which is $\left(S_{A}\right)$-map. We say that such extension is an $\left(S_{C, A}\right)$-extension of $g$.

It is easy to see that if $r: M(A) \rightarrow \mathcal{O}(Y)$ is a *representation of $M(A)$ on Hilbert space $Y$, then there is a $r$-stable space $Z \subset Y$ (i.e. $Z$ is a closed subspace of $Y$ such that $r_{a}(Z) \subset Z$ for all $a \in M(A)$ ) such that $r(A)\left(Z^{\perp}\right)=\{0\}$ and $r(A)$ acts on $Z$ non-degenerately. Based on this fact, we can prove:

Lemma 4.1. Let $f: M(A) \rightarrow \mathcal{O}(X)$ be a completely bounded map, then $f$ is a $\left(S_{A}\right)$-map on M(A) if and only if $\mathrm{f}$ has a representation $(W, V, r, Y)$ (see Definition 4.1) such that $\left.r\right|_{A}: A \rightarrow \mathcal{O}(Y)$ is non-degenerate. If $A$ and $X$ are separable, then $Y$ can be chosen to be separable.

proof. Suppose that $f$ is a $\left(S_{A}\right)$-map on $M(A)$. Let $\left(W^{\prime}, V^{\prime}, r^{\prime}, Y^{\prime}\right)$ be a non-degenerate representation of $f$ (notice that if $A$ and $X$ are separable, then $Y^{\prime}$ can be chosen to be separable [11, page 45]), then there is a $r^{\prime}$-stable space $Z \subset Y^{\prime}$ such that $r^{\prime}(A)\left(Z^{\perp}\right)=\{0\}$ and $r^{\prime}(A)$ acts on $Z$ nondegenerately. Let $E$ be the projection of $Y^{\prime}$ onto $Z$, then for any $\xi \in X$ and $a \in M(A)$, we have

$$
\begin{aligned}
f(a)(\xi) & =W^{* *} r^{\prime}(a) V^{\prime}(\xi)=W^{\prime *} r^{\prime}(a) E V^{\prime}(\xi) \\
& +W^{\prime *} r^{\prime}(a)(I-E) V^{\prime}(\xi) .
\end{aligned}
$$

Let us define $r: M(A) \rightarrow \mathcal{O}(Z)$ by $r(a)=E r^{\prime}(a) E$ for all $a \in M(A)$ (recall that $E$ is in the commuting algebra of $r^{\prime}$ because $Z$ is $r$-stable), then $r$ is a non-degenerate $*$ representation of $M(A)$ such that $\left.r\right|_{A}$ is non-degenerate. Let $\left\{a_{i}\right\}_{i \in I} \subset A$ be a norm-bounded net such that $a_{i} \rightarrow a$ strictly, then $r\left(a_{i}\right) \rightarrow r(a)$ in strong*-operator topology of $\mathcal{O}(Z)$ provided $i \rightarrow \infty$. Hence we have

$$
\begin{aligned}
f(a)(\xi) & =\lim _{i \rightarrow \infty} f\left(a_{i}\right)(\xi) \\
& =W^{\prime *} \operatorname{Er}(a) E V^{\prime}(\xi) \quad(\xi \in X) .
\end{aligned}
$$

If we define $W=E W^{\prime}$ and $V=E V^{\prime},(21)$ implies that

$$
f(a)=W^{*} r(a) V \quad(a \in M(A)) .
$$

Therefore, if we set $Y=Z$, then $(W, V, r, Y)$ is a representation of $f$ such that $r(A)$ acts on $Z$ non-degenerately.

Conversely, suppose that $f$ has a non-degenerate representation $(W, V, r, Y)$ such that $\left.r\right|_{A}$ is non-degenerate *-representation of $A$ on $Y$. Let $\left\{a_{i}\right\}_{i \in I}$ be a norm-bounded net of elements of $A$ such that $a_{i} \rightarrow a$ in $M(A)$ strictly, we have

$$
\lim _{i \rightarrow \infty} f\left(a_{i}\right)(\xi)=f(a)(\xi) \quad(\xi \in X) .
$$

On the other hand, by the same argument we have

$$
f(a)^{*}(\xi)=\lim _{i \rightarrow \infty} f\left(a_{i}\right)^{*}(\xi) \quad(\xi \in X) .
$$

Combining these two qualities, we conclude that $f\left(a_{i}\right) \rightarrow$ $f(a)$ in strong* topology of $\mathcal{O}(X)$. Our proof is complete.

Corollary 4.1. If $f: A \rightarrow \mathcal{O}(X)$ is a completely bounded map, then $f$ has a unique $\left(S_{A, A}\right)$-extension $\tilde{f}$ on $M(A)$, which satisfies

$$
\widetilde{f}(a)=\lim _{i \rightarrow \infty} f\left(a_{i}\right),
$$

provided that $a \in M(A)$ and $\left\{a_{i}\right\}_{i \in I} \subset A$ is a norm-bounded net converging to $a$ strictly, and the right side limit is in strong*-topology of $\mathcal{O}(X)$.

proof. Let $(W, V, r, Y)$ be a non-degenerate representation of $f$, then we extend $r$ to the unique non-degenerate * representation of $M(A)$ which we still denote by $r$, and define $\widetilde{f}: M(A) \rightarrow \mathcal{O}(Y)$ by

$$
\widetilde{f}(a)=W^{*} r(a) V \quad(a \in M(A)) .
$$

Then $\tilde{f}$ is an extension of $f$, and by Proposition $4.1, \tilde{f}$ is a $\left(S_{A}\right)$-map on $M(A)$. Furthermore, since $A$ is dense in $M(A)$ in the strict topology, (22) is obvious.

The following corollary contains an alternative and elementary proof of non-unital version of Stinepring's Theorem:

Corollary 4.2. Let $f: A \rightarrow \mathcal{O}(X)$ be a completely positive 
map, then

(i) f has a unique $\left(S_{A, A}\right)$-extension $\tilde{f}$ which is completely positive;

(ii) $\mathrm{f}$ has a representation $(V, R)$ (see the second paragraph of Definition 4.1);

(iii) let $\left\{a_{i}\right\}_{i \in I}$ be any approximate unit of $A$ which satisfies $\left\|a_{i}\right\| \leq 1$, then we have

$$
\|f\|_{\mathrm{cb}}=\lim _{i \rightarrow \infty}\left\|f\left(a_{i}\right)\right\|=\|\tilde{f}\|_{\mathrm{cb}} .
$$

proof $(i)$ : By Corollary 4.1, $f$ has a unique $\left(S_{A, A}\right)$ extension on $M(A)$. Furthermore, by (22), $\widetilde{f}$ is completely positive, $(i)$ is proved.

(ii): Since $M(A)$ is unital, we can apply the unital version Stinepring's Theorem to $\tilde{f}$ to get a representation $(V, R)$ of $\tilde{f}$. Therefore $\left(V,\left.R\right|_{A}\right)$ is a representation of $f,(i i)$ is proved.

(iii): Let $\left\{a_{i}\right\}_{i \in I}$ be an approximate unit of $A$. Since $\tilde{f}$ is $\left(S_{A}\right)$-map on $M(A)$ and completely positive, we have

$$
\tilde{f}\left(1_{M(A)}\right)(\xi)=\lim _{i \rightarrow \infty} \tilde{f}\left(a_{i}\right)(\xi) \quad(\xi \in X) .
$$

It implies that

$$
\left\|\tilde{f}\left(1_{M(A)}\right)\right\| \leq \underline{\lim }_{i \rightarrow \infty}\left\|\tilde{f}\left(a_{i}\right)\right\|
$$

Combining (24) with $\left\|\widetilde{f}\left(a_{i}\right)\right\| \leq\|\widetilde{f}\|_{\mathrm{cb}}=\left\|\widetilde{f}\left(1_{M(A)}\right)\right\|$, we have $\|\widetilde{f}\|_{\mathrm{cb}}=\|f\|_{\mathrm{cb}}$. Our proof is complete.

Corollary 4.3. Assume that $C$ is a $C^{*}$-subalgebra of $A$. If $f: C \rightarrow \mathcal{O}(X)$ is a completely bounded map, then $\mathrm{f}$ is a $\left(S_{C, A}\right)$-extendable map. Furthermore, if $f$ is completely positive, the extension of $f$ can be chosen to be completely positive.

proof. Since we can extend $f$ to a completely bounded map from $A$ into $\mathcal{O}(Y)$, then by Corollary 4.1, we conclude that $f$ has a $\left(S_{C, A}\right)$-extension.

Now suppose that $f$ is completely positive. By Corollary 4.2, we have a non-degenerate representation $(V, r)$ of $f$. Let us extend $r$ to a non-degenerate $*$-representation $\widetilde{r}$ of the $C^{*}$ algebra $C^{\prime}=\left\{b+t 1_{M(A)}: b \in C, t \in \mathbb{C}\right\}^{-\|\cdot\|_{M(A)}}$ (to see the existence of the extension, notice that $C$ is a closed ${ }^{*}$-ideal of $\left.C^{\prime}\right)$. We define $g^{\prime}: C^{\prime} \rightarrow \mathcal{O}(X)$ by

$$
g^{\prime}(a)=V^{*} \widetilde{r}(a) V \quad\left(a \in C^{\prime}\right)
$$

Then $g^{\prime}$ is completely positive. Since $C^{\prime}$ is a $C^{*}$-subalgebra of $A^{\prime}=\left\{a+t 1_{M(A)}: a \in A ; t \in \mathbb{C}\right\}^{-\|\cdot\|_{M(A)}}$ such that $C^{\prime}$ contains the unit of $A^{\prime}$ (i.e $1_{M(A)}$ ), we can extend $g^{\prime}$ to a completely positive map $g$ from $A^{\prime}$ into $\mathcal{O}(X) . g \mid A$ is a completely positive map which extends $f$, by Corollary 4.2, our proof is complete.

Combing Corollary 4.1 and Corollary 4.3 we have:

Corollary 4.4. Let $C \subset A$ be a $C^{*}$-subalgebra of $A$ containing an approximate unit $\left\{b_{i}\right\}_{i \in I}$ of $A$ which satisfies $\left\|b_{i}\right\| \leq 1(i \in I)$. If $f: C \rightarrow \mathcal{O}(X)$ is a completely positive map, we have

$$
\|f\|_{\mathrm{cb}}=\lim _{i \rightarrow \infty}\left\|f\left(b_{i}\right)\right\|
$$

proof. Let $\tilde{f}$ be a $\left(S_{C, A}\right)$-extension of $f$ on $M(A)$ (the existence is proved by Corollary 4.3), then by Corollary 4.2, our proof is complete.

In the rest of this section, we will apply our definitions and propositions to $C^{*}$-algebras $A=\mathcal{O}_{c}\left(\mathfrak{L}_{2}(G)\right) \otimes \rho_{\text {int }}\left(C^{*}(\mathfrak{B})\right)$ and $C=\mathfrak{E}(\rho, \mathfrak{B})$ (see Lemma 3.2 and Lemma 3.3). Recall that if $G$ is non-discrete, both $A$ and $C$ are separable $C^{*}$ algebra by Remark 2.6.

Proposition 4.1. Let us denote $\mathcal{O}_{c}\left(\mathfrak{L}_{2}(G)\right) \otimes \rho_{\text {int }}\left(C^{*}(\mathfrak{B})\right)=$ $A$ and $\mathfrak{E}(\rho, \mathfrak{B})=C$ (see Lemma 3.2). Recall that, from Lemma 3.3, $C \subset M(A)$. Let $f: C \rightarrow \mathcal{O}\left(\mathfrak{L}_{2}(G, X(\rho))\right)$ (See Remark 2.7 ) be a completely bounded map. Consider the conditions:

(i) $f$ is $\left(S_{C, A}\right)$-extendable;

(ii) $f$ has a non-degenerate representation $(W, V, R)$ such that

$$
R: C \rightarrow \mathfrak{E}(r, \mathfrak{B}), T_{k}^{\rho} \mapsto T_{k}^{r} \quad\left(k \in \mathfrak{L}_{2}(\mathfrak{B} \times \mathfrak{B})\right)
$$

for some $*$-representation $r$ of $\mathfrak{B}$ which is weakly contained in $\rho$.

Then we have $(i i) \Rightarrow(i)$, and if either $G$ is discrete or $X(\rho)$ is separable we have $(i) \Rightarrow(i i)$. Furthermore, if $f$ is completely positive, we can choose $V=W$.

proof. $\quad(i) \Rightarrow($ ii $)$ if $X(\rho)$ is separable: We denote the $S_{C, A}$-extension of $f$ on $M(A)$ still by $f$. By Lemma $4.1, f$ has a non-degenerate representation $\left(V^{\prime}, W^{\prime}, S, Y\right)$, such that $\left.S\right|_{A}$ is a non-degenerate $*$-representation of $A$, and by Lemma 4.1 again, $Y$ can be chosen to be separable. By [10, Lemma 2.5], there is a separable Hilbert space $Z$, unitary operators $U: Y \rightarrow \mathfrak{L}_{2}(G, Z)$ and a non-degenerate $*$-representation $r^{\prime}: \rho_{\text {int }}\left(C^{*}(\mathfrak{B})\right) \rightarrow \mathcal{O}\left(\mathfrak{L}_{2}(G, Z)\right)$, such that

$$
S\left(T_{h}^{\rho_{\mathrm{int}}}\right)=U^{*} T_{h}^{r^{\prime} \circ \rho_{\mathrm{int}}} U \quad\left(h \in \mathfrak{L}_{2}\left(G \times G, C^{*}(\mathfrak{B})\right) .\right.
$$

It is clear that $r^{\prime} \circ \rho_{\text {int }}: C^{*}(\mathfrak{B}) \rightarrow \mathcal{O}(Z)$ is non-degenerate, thus $r^{\prime} \circ \rho_{\text {int }}$ is the integrated form of a $*$-representation of $\mathfrak{B}$ on $Z$ which we denote by $r$. Then $r$ is weakly contained in $\rho$. For fixed $k \in \mathfrak{L}(\mathfrak{B} \times \mathfrak{B})$, by Lemma 3.3, we have

$$
\begin{aligned}
\left(U^{*} T_{k}^{r} U\right) \circ S\left(T_{h}^{\rho_{\mathrm{int}}}\right) & =\left(U^{*} T_{k}^{r} U\right) \circ\left(U^{*} T_{h}^{r^{\prime} \circ \rho_{\mathrm{int}}} U\right) \\
& =U^{*} T_{k \star h}^{r^{\prime} \circ \rho_{\mathrm{int}}} U \quad(h \in \mathfrak{K}(\mathfrak{B})) .
\end{aligned}
$$

On the other hand, by Lemma 3.3 again, we have

$$
S\left(T_{k}^{\rho}\right) S\left(T_{h}^{\rho_{\text {int }}}\right)=S\left(T_{k \star h}^{\rho_{\text {int }}}\right)=U^{*} T_{k \star h}^{r^{\prime} \circ \rho_{\text {int }}} U \quad(h \in \mathfrak{K}(\mathfrak{B})) .
$$

Since $\mathfrak{K}(\mathfrak{B})$ is dense in $A$ and $\left.S\right|_{A}$ is non-degenerate *representation of $A$, we conclude that

$$
S\left(T_{k}^{\rho}\right)=U^{*} T_{k}^{r} U \quad(k \in \mathfrak{L}(\mathfrak{B} \times \mathfrak{B})) .
$$

Now let $W=U W^{\prime}, V=U V^{\prime}$, and define a * homomorphism $R: \mathfrak{E}(\rho, \mathfrak{B}) \rightarrow \mathfrak{E}(r, \mathfrak{B})$ by $R\left(T_{k}^{\rho}\right)=T_{k}^{r}(k \in$ $\left.\mathfrak{L}_{2}(\mathfrak{B} \times \mathfrak{B})\right)\left(\right.$ see Lemma 3.4). Since $\left\{T_{k}^{\rho}: k \in \mathfrak{L}_{2}(\mathfrak{B} \times \mathfrak{B})\right\}$ is dense in $\mathfrak{E}(\rho, \mathfrak{B}),(25)$ implies that

$$
f(a)=W^{*} R(a) V \quad(a \in \mathfrak{E}(\rho, \mathfrak{B})) .
$$


$(i) \Rightarrow(i i)$ is proved.

By the similar argument, we can prove that $(i) \Rightarrow(i i)$ if $G$ is discrete.

$(i i) \Rightarrow(i)$ : Since $r$ is weekly contained in $\rho$, then by Lemma 3.4, the following map

$$
S: T_{h}^{\rho_{\text {int }}} \mapsto T_{h}^{r_{\text {int }}} \quad\left(h \in \mathfrak{L}_{2}\left(G \times G, C^{*}(\mathfrak{B})\right)\right)
$$

is $*$-homomorphism from $A$ onto $\mathcal{O}_{c}\left(\mathfrak{L}_{2}(G)\right) \otimes r_{\text {int }}\left(C^{*}(\mathfrak{B})\right)$. We define $F: M(A) \rightarrow \mathcal{O}\left(\mathfrak{L}_{2}(G, X)\right)$ by

$$
F(a)=W^{*} S(a) V \quad(a \in M(A)) .
$$

Since $S$ is non-degenerate, by Lemma $4.1, F$ is a $\left(S_{A}\right)$-map on $M(A)$ and an extension of $f$. Our proof is complete.

For any $a \in \mathfrak{L}_{\infty}(G)$, we define $\bar{a} \in \mathfrak{L}_{\infty}(G)$ by $\bar{a}(s)=\overline{a(s)}$ for all $s \in G$. We associate an operator $M_{a}: \mathfrak{L}_{2}(G) \rightarrow \mathfrak{L}_{2}(G)$ defined by

$$
M_{a}(\xi)(s)=a(s) \xi(s) \quad\left(\xi \in \mathfrak{L}_{2}(G) ; s \in G\right) .
$$

It is clear that $M_{a}^{*}=M_{\bar{a}}$, thus $\mathfrak{M}(G)=\left\{M_{a}: a \in\right.$ $\left.\mathfrak{L}_{\infty}(G)\right\}$ is a concrete $C^{*}$-algebra acting on the Hilbert space $\mathfrak{L}_{2}(G)$.

For $k \in \mathfrak{L}_{2}(\mathfrak{B} \times \mathfrak{B})$, we define

$$
a \cdot k(s, t)=a(s) k(s, t), k \cdot a(s, t)=k(s, t) a(t),
$$

for $a \in \mathfrak{L}_{\infty}(G)$ and $s, t \in G$. Then it is easy to verify that $a \cdot k$, $k \cdot a$ are in $\mathfrak{L}_{2}(\mathfrak{B} \times \mathfrak{B})$. Furthermore, for any $k \in \mathfrak{L}_{2}(\mathfrak{B} \times \mathfrak{B})$ and $a, b \in \mathfrak{L}_{\infty}(G)$,

$$
\left(M_{a} \otimes I_{X(\rho)}\right) T_{k}^{\rho}\left(M_{b} \otimes I_{X(\rho)}\right)=T_{a \cdot k \cdot b}^{\rho} .
$$

If $\Phi$ is a $\operatorname{Schur}(\mathfrak{B}, \rho)$-multiplier, we have

$$
\begin{aligned}
& S_{\rho, \Phi}\left(\left(M_{a} \otimes I_{X(\rho)}\right) x\left(M_{b} \otimes X(\rho)\right)\right) \\
= & \left(M_{a} \otimes I_{X(\rho)}\right) S_{\rho, \Phi}(x)\left(M_{a} \otimes I_{X(\rho)}\right)
\end{aligned}
$$

for all $x \in \mathfrak{E}(\rho, \mathfrak{B})$ and $a, b \in \mathfrak{L}_{\infty}(G)$.

Theorem 4.1. Let us denote $\mathcal{O}_{c}\left(\mathfrak{L}_{2}(G)\right) \otimes \rho_{\text {int }}\left(C^{*}(\mathfrak{B})\right)=A$ and $\mathfrak{E}(\rho, \mathfrak{B})=C$. Recall that, from Lemma 3.3, $C \subset M(A)$. If $\Phi: D \rightarrow D$ be a multiplier of $\mathfrak{B} \times \mathfrak{B}$, and consider the following two statements:

(i) $\Phi$ is a $\operatorname{Schur}(\mathfrak{B}, \rho)$-multiplier such that $S_{\rho, \Phi}$ is $\left(S_{C, A}\right)$ extendable;

(ii) There is non-degenerate *-representation $r: B \rightarrow$ $\mathcal{O}(Y)$ of $\mathfrak{B}$ on a Hilbert space $Y$ (or denoted by $Y(r)$ ) which is weakly contained in $\rho$, and

$$
V, W \in \mathfrak{L}_{\infty}(G, \mathcal{O}(X, Y))
$$

such that

$\rho\left(\Phi((x, y ; a))=W^{*}(x) r_{D}((x, y ; a)) V(y) \quad\left((x, y ; a) \in D_{x, y}\right)\right.$

(see Definition 2.2 for the notations) for almost all $(x, y) \in$ $G \times G$. Then we have $(i i) \Rightarrow(i)$, and if either $X(\rho)$ is separable or $G$ is discrete then we have $(i) \Rightarrow(i i)$.
(If $G$ is discrete, then by [7, VIII.16.11], for any *representation $\rho$ of $\mathfrak{B}$ we always have $\rho(B) \subset \rho_{\text {int }}\left(C^{*}(\mathfrak{B})\right)$. Then we have

$$
\mathfrak{E}(\rho, \mathfrak{B}) \subset \mathcal{O}_{c}\left(\mathfrak{L}_{2}(G)\right) \otimes \rho_{\text {int }}\left(C^{*}(\mathfrak{B})\right) .
$$

Corollary 4.3 implies that every completely bounded multipliers is $\left(S_{C, A}\right)$-extendable. Therefore, we can remove ' $S_{\rho, \Phi}$ is $\left(S_{C, A}\right)$-extendable' in $(i)$, and (i) and (ii) are always equivalent.)

proof. We prove that $(i) \Rightarrow(i i)$ if $X(\rho)$ is separable, the argument in the case that $G$ is discrete is similar. Since $S_{\rho, \Phi}$ is $\left(S_{C, A}\right)$-extendable, by Proposition 4.1 there are separable Hilbert space $Y$, non-degenerate $*$-representation $r: B \rightarrow$ $\mathcal{O}(Y)$ which is weakly contained in $\rho$, and bounded operators $V_{0}, W_{0}$ in $\mathcal{O}\left(\mathfrak{L}_{2}(G, X), \mathfrak{L}_{2}(G, Y)\right)$ such that

$$
S_{\rho, \Phi}\left(T_{k}^{\rho}\right)=W_{0}^{*} T_{k}^{r} V_{0} \quad\left(k \in \mathfrak{L}_{2}(\mathfrak{B} \times \mathfrak{B})\right) .
$$

Let $\mathcal{C}=\overline{\left[\left\{T_{k}^{r} V_{0} \mathfrak{L}_{2}(G, X): k \in \mathfrak{L}_{2}(\mathfrak{B} \times \mathfrak{B})\right\}\right]} \subset \mathfrak{L}_{2}(G, Y)$, and $E$ the prjection of $\mathfrak{L}_{2}(G, Y)$ onto $\mathcal{C}$. Since $\mathcal{C}$ is invariant under the concrete $C^{*}$-algebra $\mathfrak{E}(r, \mathfrak{B})$, we have $E T_{k}^{r}=T_{k}^{r} E$ for all $k \in \mathfrak{L}_{2}(\mathfrak{B} \times \mathfrak{B})$.

For any $d \in \mathfrak{L}_{\infty}(G)$ and $k \in \mathfrak{L}_{2}(\mathfrak{B} \times \mathfrak{B})$, we have

$$
\left(M_{d} \otimes I_{Y}\right)^{*} T_{k}^{r}=T_{\bar{d} \cdot k}^{r} \quad\left(d \in \mathfrak{L}_{\infty}(G) ; k \in \mathfrak{L}_{2}(\mathfrak{B} \times \mathfrak{B})\right),
$$

and

$$
W_{0}^{*} T_{\bar{d} \cdot k} V_{0}=\left(M_{d}^{*} \otimes I_{X}\right) W_{0}^{*} T_{k}^{r} V_{0} .
$$

Let $W=E W_{0}$, combining (28) and (27), we have

$$
\left\langle T_{k}^{r} V_{0} \xi,\left(M_{d} \otimes I_{Y}\right) W \eta\right\rangle=\left\langle T_{k}^{r} V_{0} \xi, W\left(M_{d} \otimes I_{X}\right) \eta\right\rangle
$$

for all $k \in \mathfrak{L}_{2}(\mathfrak{B})$ and $\xi, \eta \in \mathfrak{L}_{2}(G, X)$. We conclude that

$$
E\left(M_{d} \otimes I_{Y}\right) W=E W\left(M_{d} \otimes I_{X}\right) \quad\left(d \in \mathfrak{L}_{\infty}(G)\right) .
$$

Furthermore, since $\mathcal{C}$ is stable under the action of the $C^{*}$ algebra $\left\{M_{d} \otimes I_{Y}: d \in \mathfrak{L}_{\infty}(G)\right\}$, we have

$$
E\left(M_{d} \otimes I_{Y}\right)=\left(M_{d} \otimes I_{Y}\right) E \quad\left(d \in \mathfrak{L}_{\infty}(G)\right) .
$$

We conclude that

$$
\left(M_{d} \otimes I_{Y}\right) W=W\left(M_{d} \otimes I_{X}\right) \quad\left(d \in \mathfrak{L}_{\infty}(G)\right) .
$$

It follows that $W \in \mathfrak{L}_{\infty}(G, \mathcal{O}(X, Y))$. By the same argument we can replace $V_{0}$ by an operator $V \in$ $\mathfrak{L}_{\infty}(G, \mathcal{O}(X, Y))$. By the same argument of the proof of [10, Theorem 2.6], we have, for any $k \in \mathfrak{L}_{2}(\mathfrak{B} \times \mathfrak{B})$,

$$
\begin{array}{r}
\rho_{D}(\Phi(k(x, y)))=W(x)^{*} r_{D}(k(x, y)) V(y) \\
\left(k \in \mathfrak{L}_{2}(\mathfrak{B} \times \mathfrak{B})\right),
\end{array}
$$

for almost all $(x, y) \in G \times G$.

Since $\mathfrak{B} \times \mathfrak{B}$ is second-countable Banach bundle over second countable locally compact space $G \times G, \mathfrak{L}(\mathfrak{B} \times \mathfrak{B})$ 
is separable. Let $\left\{k_{n}\right\}_{n \in \mathbb{N}} \subset \mathfrak{L}(\mathfrak{B} \times \mathfrak{B})$ be a countable dense subset of $\mathfrak{L}(\mathfrak{B} \times \mathfrak{B})$. In particular, for fixed $x, y \in G$, the set $\left\{k_{n}(x, y): n \in \mathbb{N}\right\}$ is dense in $D_{x, y}$. On the other hand, for each $n \in \mathbb{N}$ we define

$$
\begin{aligned}
N_{n} & =\left\{(x, y) \in G \times G: W(x)^{*} r_{D}\left(k_{n}(x, y)\right) V(y)\right. \\
& \left.\neq \rho_{D}\left(\Phi\left(k_{n}(x, y)\right)\right)\right\} .
\end{aligned}
$$

Then $N=\bigcup_{n \in \mathbb{N}} N_{n}$ is a null-subset of $G \times G$. If $(x, y) \in$ $(G \times G) \backslash N$, then for any $a_{x, y} \in D_{x, y}$, since there is a sequence $\left\{k_{n_{i}}\right\}_{i \in \mathbb{N}} \subset\left\{k_{n}\right\}_{n \in \mathbb{N}}$ such that $k_{n_{i}}(x, y) \rightarrow a_{x, y}$, we have

$$
\rho_{D}\left(\Phi\left(a_{x, y}\right)\right)=W(x)^{*} r_{D}\left(a_{x, y}\right) V(y) .
$$

The proof of $(i) \Rightarrow(i i)$ is complete.

$(i i) \Rightarrow(i):$ For any $k \in \mathfrak{L}_{2}(\mathfrak{B} \times \mathfrak{B})$, we have

$$
\begin{aligned}
\left\langle S_{\rho, \Phi}\left(T_{k}^{\rho}\right) \xi, \eta\right\rangle & \left.=\int_{G} W(x)^{*} r_{D}(k(x, y)) V(y) \xi(y), \eta(x)\right\rangle d x d y \\
& =\left\langle\left(W^{*} T_{k}^{*} V\right) \xi, \eta\right\rangle \quad\left(\xi, \eta \in \mathfrak{L}_{2}(G, X)\right) .
\end{aligned}
$$

Hence,

$$
S_{\rho, \Phi}\left(T_{k}^{\rho}\right)=W^{*} T_{k}^{r} V
$$

Since $r$ is weakly contained in $\rho$, by Proposition 4.1, our proof is complete.

\section{Herz-Schur Multipliers}

In this section, we fix $\rho: B \rightarrow \mathcal{O}(X)$ to be a non-degenerate *-representation of $\mathfrak{B}$. We remind the reader that $\rho_{\text {int }}$ is the integrated form of $\rho$.

Let $\Psi: B \rightarrow B$ be a multiplier of $\mathfrak{B}$ (see Definition 3.2). For each $f \in \mathfrak{L}(\mathfrak{B})$, we define $\Psi \cdot f \in \mathfrak{L}(\mathfrak{B})$ by

$$
\Psi \cdot f(x)=\Psi(f(x)) \quad(x \in G) .
$$

Definition 5.1. Let $\varsigma: B \rightarrow \mathcal{O}(Y)$ be a $*$-representation of $\mathfrak{B}$ on a Hilbert space $Y$ such that $\varsigma \mid B_{e}$ is faithful. A multiplier $\Psi: B \rightarrow B$ of $\mathfrak{B}$ is called $(\varsigma, \mathfrak{B})$-multiplier if the map $S_{\Psi}^{\varsigma}$, which is defined on $\left\{\varsigma_{\text {int }}(f): f \in \mathfrak{L}_{1}(\mathfrak{B})\right\} \subset \mathcal{O}(Y)$ by

$$
S_{\Psi}^{\varsigma}\left(\varsigma_{\text {int }}(f)\right)=\varsigma_{\text {int }}(\Psi \cdot f) \quad\left(f \in \mathfrak{L}_{1}(\mathfrak{B})\right),
$$

is completely bounded. In this case, $S_{\Psi}^{\varsigma}$ can be extended to a completely bounded map on $\left\{\varsigma_{\text {int }}(f): f \in \mathfrak{L}_{1}(\mathfrak{B})\right\}^{-\mathcal{O}(Y)}$ which we still denote by $S_{\Psi}^{\varsigma}$.

Remark 5.1. Notice that if $r$ and $\varsigma$ are weakly equivalent *representation of $\mathfrak{B}$, then it is easy to see that $\Psi$ is $(\varsigma, \mathfrak{B})$ multiplier if and only if $\Psi$ is $(r, \mathfrak{B})$-multiplier, and in this case

$$
\left\|S_{\Psi}^{\varsigma}\right\|_{\mathrm{cb}}=\left\|S_{\Psi}^{r}\right\|_{\mathrm{cb}}
$$

We use the symbol $\lambda_{\mathfrak{B}}$ to denote the regular *-representation of $C^{*}(\mathfrak{B})$, and we denote the reduced $C^{*}$-algebra of $\mathfrak{B}$, i.e $C^{*}(\mathfrak{B}) / \operatorname{Ker}\left(\left(\lambda_{\mathfrak{B}}\right)_{\text {int }}\right)$, by $C_{\text {Red }}^{*}(\mathfrak{B})$. For the details about regular $*$-representationand the reduced $C^{*}$-algebra, we refer the reader to [5].
Let $\lambda: G \rightarrow \mathcal{O}\left(\mathfrak{L}_{2}(G)\right)$ be the left regular representation of $G$. By [7, VIII.9.16], we can form a ${ }^{*}$-representation $\rho \otimes \lambda$ of $\mathfrak{B}$ on $\mathfrak{L}_{2}(G) \otimes X(\rho)$, which is weakly equivalent to $\lambda_{\mathfrak{B}}$ (by [5]), defined by

$$
(\rho \otimes \lambda)_{b}=\lambda_{\pi(b)} \otimes \rho_{b} \quad(b \in B) .
$$

From [5] we can identify $C_{\text {Red }}^{*}(\mathfrak{B})$ with

$$
\left\{(\rho \otimes \lambda)_{\text {int }}(f): f \in \mathfrak{L}_{1}(\mathfrak{B})\right\}^{-\mathcal{O}\left(\mathfrak{L}_{2}(G, X)\right)} .
$$

Recall that, for any $f \in \mathfrak{L}(\mathfrak{B})$ and $\xi \in \mathfrak{L}_{2}(G, X)$, we have

$$
(\rho \otimes \lambda)_{\mathrm{int}}(f)(\xi)(x)=\int_{G} \rho(f(y))\left(\xi\left(y^{-1} x\right)\right) d y,
$$

for almost all $x \in G$.

Definition 5.2. We call a multiplier $\Psi: B \rightarrow B$ of $\mathfrak{B}$ Herz-Schur multiplier of $\mathfrak{B}$ if $\Psi$ is $(\rho \otimes \lambda, \mathfrak{B})$-multiplier. Furthermore, if $S_{\Psi}^{\rho \otimes \lambda}$ is completely positive, we call $\Psi$ completely positive Herz-Schur multiplier of $\mathfrak{B}$.

If $\Psi$ is Herz-Schur multiplier, we define

$$
\|\Psi\|_{\mathfrak{H} \cdot \mathfrak{S}}=\left\|S_{\Psi}^{\rho \otimes \lambda}\right\|_{\mathrm{cb}}
$$

Remark 5.2. By Remark 5.1, Definition 5.2 is independent of the choice of *-representation of $\rho: B \rightarrow \mathcal{O}(X)$ satisfying that $\left.\rho\right|_{B_{e}}$ is faithful.

Therefore, if $\Psi$ is a Herz-Schur multiplier, $S_{\Psi}^{\rho \otimes \lambda}$ can be extended to $\left\{(\rho \otimes \lambda)_{\text {int }}(f): f \in \mathfrak{L}_{1}(\mathfrak{B})\right\}^{-\mathcal{O}\left(\mathfrak{L}_{2}(G, X)\right)}$. We $\operatorname{regard} S_{\Psi}^{\rho \otimes \lambda}$ is completely bounded map defined on $C_{\text {Red }}^{*}(\mathfrak{B})$.

In the following, if $\Psi$ is a Herz-Schur multiplier, we will denote $S_{\Psi}^{\rho \otimes \lambda}$ briefly by $S_{\Psi}$.

Proposition 5.1. Let $\varsigma: B \rightarrow \mathcal{O}(Y)$ be a $*$-representation of $\mathfrak{B}$, and $\Psi: B \rightarrow B$ a multiplier of $\mathfrak{B}$, the following are equivalent:

(i) $\Psi$ is a $(\varsigma, \mathfrak{B})$-multiplier;

(ii) There is a non-degenerate $*$-representation $r: B \rightarrow$ $\mathcal{O}(Z)$ on Hilbert space $Z$ which is weakly contained in $\varsigma$, and bounded operators $V, W \in \mathcal{O}(Y, Z)$ (here $Y=X(\varsigma)$ ) such that

$$
\varsigma\left(\Psi\left(a_{x}\right)\right)=W^{*} r\left(a_{x}\right) V \quad\left(a_{x} \in B_{x}\right)
$$

for all $x \in G$ and $\left\|S_{\Psi}^{\varsigma}\right\|_{\mathrm{cb}}=\|V\|\|W\|$.

proof. $\quad(i) \Rightarrow(i i) \quad$ : Since $S_{\Psi}^{\varsigma}: \varsigma_{\text {int }}\left(C^{*}(\mathfrak{B})\right) \rightarrow$ $\mathcal{O}(Z)$ is a completely bounded map, let $(W, V, R, Z)$ be its representation.

Therefore, let $r: B \rightarrow \mathcal{O}(Z)$ be the $*$-representation of $\mathfrak{B}$ whose integrated form is $R \circ \varsigma_{\text {int }}$, we have

$$
\begin{aligned}
S_{\Psi}^{\varsigma}\left(\varsigma_{\text {int }}(f)\right) & =W^{*} r_{\operatorname{int}}(f) V \\
& =W^{*} \int_{G} r(f(x)) d x V \quad\left(f \in \mathfrak{L}_{1}(\mathfrak{B})\right) .
\end{aligned}
$$

Now let $y \in G$ and $a_{y} \in B_{y}$, and $\left\{g_{i}\right\}_{i \in I} \subset \mathfrak{L}(G)$ a net such that $\operatorname{supp}\left(g_{i}\right) \rightarrow y, g_{i} \geq 0$ and $\int_{G} g_{i}(x) d x=1$. 
Furthermore, let $f \in \mathfrak{L}(\mathfrak{B})$ such that $f(y)=a_{y}$, we have

$$
S_{\Psi}^{\varsigma}\left(\varsigma_{\text {int }}\left(g_{i} f\right)\right) \underset{\text { strong operator }}{\longrightarrow} \varsigma(\Psi(f(y)))=\varsigma\left(\Psi\left(a_{y}\right)\right) .
$$

On the other hand,

$$
\begin{gathered}
W^{*}\left(\int_{G} \varsigma\left(g_{i}(x) f(x)\right) d x\right) V \underset{\text { strong operator }}{\longrightarrow} W^{*} r(f(y)) V \\
=W^{*} r\left(a_{y}\right) V .
\end{gathered}
$$

Therefore, we have

$$
\varsigma\left(\Psi\left(a_{y}\right)\right)=W^{*} r\left(a_{y}\right) V \quad\left(y \in G ; a_{y} \in B_{y}\right)
$$

$(i i) \Rightarrow(i)$ : Since $r$ is weakly contained in $\varsigma$, then we have a *-homomorphism $R: \varsigma_{\text {int }}\left(C^{*}(\mathfrak{B})\right) \rightarrow r_{\text {int }}\left(C^{*}(\mathfrak{B})\right)$ satisfying

$$
R\left(\varsigma_{\text {int }}(f)\right)=r_{\text {int }}(f) \quad\left(f \in \mathfrak{L}_{1}(\mathfrak{B})\right) .
$$

Therefore, for arbitrary $\xi \in Y$, we have

$$
\varsigma_{\text {int }}(\Psi \cdot f)(\xi)=W^{*} R\left(\varsigma_{\text {int }}(f)\right) V(\xi) \quad\left(f \in \mathfrak{L}_{1}(\mathfrak{B})\right) .
$$

So $\varsigma_{\text {int }}(\Psi \cdot f)=W^{*} R\left(\varsigma_{\text {int }}(f)\right) V$ for all $f \in \mathfrak{L}_{1}(\mathfrak{B})$, and we conclude that $S_{\Psi}^{\varsigma}$ is completely bounded.

Let $\Psi: B \rightarrow B$ be a multiplier of $\mathfrak{B}$, then we define a map $N(\Psi): D \rightarrow D$ (recall that $D$ is the bundle space of $\mathfrak{B} \times \mathfrak{B}$ ) by

$$
\begin{aligned}
& N(\Psi)((x, y ; a))=(x, y ; \Psi(a)) \\
& \left(x, y \in G ;(x, y ; a) \in D_{x, y}\right) .
\end{aligned}
$$

It is routine to check that $N(\Psi)$ satisfies $(i)-(i i)$ of Definition 3.2, thus $N(\Psi)$ is a multiplier of $\mathfrak{B} \times \mathfrak{B}$.

Theorem 5.1. Suppose that $\rho$ weakly contains $\lambda_{\mathfrak{B}}$. Let $\Psi$ : $B \rightarrow B$ be a multiplier of $\mathfrak{B}$. The following are equivalent:

(i) $\Psi$ is (resp. completely positive) Herz-Schur multiplier of $\mathfrak{B}$;

(ii) $N(\Psi)$ is (resp. completely positive) Schur $(\rho, \mathfrak{B})$ multiplier.

If either $(i)$ or $(i i)$ holds, we have

$$
\|\Psi\|_{\mathfrak{H} . \mathfrak{S}}=\|N(\Psi)\|_{\mathfrak{S}} .
$$

proof. $\quad(i) \Rightarrow($ ii $)$ : By Proposition 5.1, we have a $*$ representation $r: B \rightarrow \mathcal{O}(Y)$ on Hilbert space $Y$ which is weakly contained in $\lambda_{\mathfrak{B}}$, and bounded operators $W_{0}, V_{0} \in$ $\mathcal{O}(X, Y)$ such that

$$
\rho \otimes \lambda\left(\Psi\left(a_{x}\right)\right)=W_{0}^{*} r\left(a_{x}\right) V_{0} \quad\left(x \in G ; a_{x} \in B_{x}\right) .
$$

By Proposition 4.1 and the same argument of $(i) \Rightarrow(i i)$ of Proposition 4.1, it is not hard to prove that $N(\Psi)$ is Schur $\left(\rho \otimes 1_{\mathcal{O}(\mathfrak{L}(G))}, \mathfrak{B}\right)$-multiplier, such that

$$
\left\|S_{\rho \otimes 1_{\mathcal{O}\left(\mathfrak{L}_{2}(G)\right)}, N(\Psi)}\right\|_{\mathrm{cb}} \leq\|V\|\|W\|=\left\|S_{\Psi}\right\|_{\mathrm{cb}} .
$$

By Proposition 3.3, $N(\Psi)$ is $(\rho, \mathfrak{B})$-multiplier.

$(i i) \Rightarrow(i)$ : By the same argument of $(i i) \Rightarrow(i)$ of the proof of [10, Theorem 3.8], $\Psi$ is Herz-Schur multiplier and satisfies

$$
\left\|(\rho \otimes \lambda)_{\mathrm{int}}(\Psi \cdot f)\right\|_{\mathrm{cb}} \leq\left\|S_{N(\Psi)}\right\|_{\mathrm{cb}} .
$$

Hence, $\left\|(\rho \otimes \lambda)_{\text {int }}(\Psi \cdot f)\right\|_{\mathrm{cb}}=\left\|S_{N(\Psi)}\right\|_{\mathrm{cb}}$. The proof is complete.

Corollary 5.1. If $\varsigma: B \rightarrow \mathcal{O}(Y)$ is a $*$-representation of $\mathfrak{B}$ which weakly contains $\lambda_{\mathfrak{B}}$, then any $(\varsigma, \mathfrak{B})$-multiplier is Herz-Schur multiplier.

proof. This is the combination of Theorem 5.1, Proposition 5.1 and Theorem 4.1.

\section{Nuclearifty of Cross-Sectional Algebra}

In this section we assume that $\mathfrak{B}$ is a Fell-bundle over a discrete group $G$, and $\rho$ a fixed ${ }^{*}$-representation of $\mathfrak{B}$ which weakly contains $\lambda_{\mathfrak{B}}$ (the regular $*$-representation of $\mathfrak{B}$ ). Recall that, under these assumptions, we have

$$
\mathfrak{E}(\rho, \mathfrak{B}) \subset \mathcal{O}_{c}\left(\mathfrak{L}_{2}(G)\right) \otimes \rho_{\text {int }}\left(C^{*}(\mathfrak{B})\right) .
$$

Therefore, by Lemma 4.3 and Theorem 4.1 we have

Corollary 6.1. Let $\Phi: D \rightarrow D$ be a multiplier of $\mathfrak{B} \times \mathfrak{B}$, then the following are equivalent:

(i) $\Phi$ is (resp. completely positive) Schur $(\mathfrak{B}, \rho)$-multiplier.

(ii) There are non-degenerate $*$-representation $r: B \rightarrow$ $\mathcal{O}(Y)$ on a Hilbert space $Y$ which is weakly contained in $\rho$, and $V, W \in \mathfrak{L}_{\infty}(G, \mathcal{O}(X, Y))$ (resp. $\left.V=W\right)$ such that

$$
\begin{aligned}
\rho_{D}(\Phi((x, y ; a)) & =W^{*}(x) r(a) V(y) \\
& \left(x, y \in G ; a \in B_{x y^{-1}}\right) .
\end{aligned}
$$

Let $\Phi: D \rightarrow D$ be a multiplier of $\mathfrak{B} \times \mathfrak{B}$, we define $\Phi_{x}^{\rho}: \rho_{D}\left(D_{x, x}\right) \rightarrow \rho_{D}\left(D_{x, x}\right)(x \in G)$ by

$$
\Phi_{x}^{\rho}\left(\rho_{D}((x, x ; a))=\rho_{D}\left(\Phi((x, x ; a)) \quad\left((x, x ; a) \in D_{x, x}\right) .\right.\right.
$$

Remark 6.1. Let us recall that each $D_{x, x}=B_{e}(x \in G)$. However it is not necessary that all $\Phi_{x}^{\rho}$ are identical.

Proposition 6.1. Let $\Phi: D \rightarrow D$ be a completely positive $\operatorname{Schur}(\rho, \mathfrak{B})$-multiplier, then each $\Phi_{x}^{\rho}$ is completely positive. Furthermore,

$$
\|\Phi\|_{\mathfrak{S}}=\sup \left\{\left\|\Phi_{x}^{\rho}\right\|_{\mathrm{cb}}: x \in G\right\} .
$$

proof. By Corollary 6.1, each $\Phi_{x}^{\rho}$ is completely positive.

Let $\left\{a_{i}\right\}_{i \in I}$ be an approximate unit of the $C^{*}$-algebra $B_{e}$ with $\left\|a_{i}\right\| \leq 1(i \in I)$. Let $\mathcal{A}$ be the collection of all finite subsets of $G$. We define the order on $\mathcal{A} \times I$ by

$$
(U, i) \leq(V, j) \Leftrightarrow U \subset V \text { and } i \leq j \quad((U, i),(V, j) \in \mathcal{A} \times I) .
$$

We define $a_{U, i}=a_{i}$ for all $(U, i) \in \mathcal{A} \times I$. It is clear that $\left\{a_{U, i}\right\}_{(U, i) \in \mathcal{A} \times I}$ is an approximate unit of $B_{e}$ since it is a subnet of $\left\{a_{i}\right\}_{i \in I}$. By [7, VIII.5.11] and [7, VIII.16.3], we have $\left\|a_{U, i} b-b\right\| \rightarrow 0$ for all $b \in C^{*}(\mathfrak{B})$. 
We define $k_{U, i} \in \mathfrak{L}(\mathfrak{B} \times \mathfrak{B})$ by

$$
\begin{aligned}
& k_{(U, i)}(x, x)=\left(x, x ; a_{U, i}\right) \quad(x \in U) ; \\
& k_{(U, i)}(y, z)=0 \quad \text { otherwise. }
\end{aligned}
$$

Hence, if $(U, i) \rightarrow \infty$, we have

$$
\lim _{(U, i) \rightarrow \infty} T_{k_{U, i}}^{\rho_{\mathrm{int}}} T_{k}^{\rho}=T_{k}^{\rho_{\mathrm{int}}} \quad(k \in \mathfrak{L}(\mathfrak{B} \times \mathfrak{B})) .
$$

Since $\left\{T_{k}^{\rho_{\text {int }}}: k \in \mathfrak{L}\left(G \times G, C^{*}(\mathfrak{B})\right)\right\}$ is dense in $\mathcal{O}_{c}\left(\mathfrak{L}_{2}(G)\right) \otimes \rho_{\text {int }}\left(C^{*}(\mathfrak{B})\right)$, (30) implies that $\left\{T_{k_{U, i}}^{\rho}\right\}$ is an approximate unit of $\mathcal{O}_{c}\left(\mathfrak{L}_{2}(G)\right) \otimes \rho_{\text {int }}\left(C^{*}(\mathfrak{B})\right)$ with norm not greater than 1 .

On the other hand, for each fixed $x \in G$, since $\rho \mid B_{e}$ is a non-degenerate $*$-representation of $B_{e}$, we conclude that $\left\|\rho_{D}\left(k_{U, i}(x, x)\right)\right\| \leq 1$, and the net

$$
\left\{\rho_{D}\left(k_{U, i}(x, x)\right)\right\}_{(U, i) \in \mathcal{A} \times I}
$$

is an approximate unit of the concrete $C^{*}$-algebra $\rho_{D}\left(D_{x, x}\right)=$ $\rho\left(B_{e}\right) \subset \mathcal{O}(X(\rho))$. By Corollary 4.4, we have

$$
\begin{aligned}
\|\Phi\|_{\mathfrak{S}} & =\lim _{(U, i) \rightarrow \infty}\left\|S_{\rho, \Phi}\left(T_{k_{(U, i)}}^{\rho}\right)\right\| \\
& =\sup \left\{\left\|\Phi_{x}^{\rho}\right\|: x \in G\right\} .
\end{aligned}
$$

For any multiplier $\Psi: B \rightarrow B$ of $\mathfrak{B}$, we define $\Psi_{x}^{\rho}$ : $\rho\left(B_{x}\right) \rightarrow \rho\left(B_{x}\right)$ by $\Psi_{x}^{\rho}\left(\rho\left(a_{x}\right)\right)=\rho\left(\Psi\left(a_{x}\right)\right)\left(a_{x} \in B_{x}\right)$ for all $x \in G$.

Corollary 6.2. If $\Psi: B \rightarrow B$ is a completely positive HerzSchur multiplier of $\mathfrak{B}$, then we have

$$
\|\Psi\|_{\mathfrak{H} . \mathfrak{S}}=\left\|\Psi_{e}^{\rho}\right\|_{\mathrm{cb}} .
$$

Definition 6.1. We call a Fell bundle $\mathfrak{B}$ nuclear if there exists a net $\{\Psi\}_{i \in I}$ of completely positive Herz-Schur multipliers of $\mathfrak{B}$ such that

i. $\left\|\Psi_{e}^{\rho}\right\|_{\mathrm{cb}} \leq 1$ for all $i \in I$;

ii. Each $\left(\Psi_{i}\right)_{x}^{\rho}$ has finite dimensional range $(x \in G ; i \in I)$; iii. $\left\|\left(\Psi_{i}\right)_{x}^{\rho}\left(\rho\left(a_{x}\right)\right)-\rho\left(a_{x}\right)\right\| \rightarrow 0$ provided $i \rightarrow \infty$ for all $s \in G$ and $a_{x} \in B_{x}$.

Assume that $A$ is nuclear $C^{*}$-algebra. Recall that there is a net $\left\{\phi_{i}\right\}_{i \in I}$ of completely positive contractive maps on $A$ such that the range of each $\phi_{i}$ is finite and $\left\|\phi_{i}(a)-a\right\| \rightarrow 0$ for all $a \in A$ provided $i \rightarrow \infty$. We call $\left\{\phi_{i}\right\}_{i \in I}$ an approximation net of $A$.

Recall that $C_{\mathrm{Red}}^{*}(\mathfrak{B})$ is *-isomorphic to $(\rho \otimes \lambda)_{\text {int }}\left(C^{*}(\mathfrak{B})\right)$. In the following we assume that $\rho: B \rightarrow \mathcal{O}(X(\rho))$ is weakly equivalent to $\lambda_{\mathfrak{B}}$. By [5, Proposition 19.3], there is a conditional expectation

$$
\mathcal{E}: \rho_{\mathrm{int}}\left(C^{*}(\mathfrak{B})\right) \otimes_{\min } C_{r}^{*}(G) \rightarrow \rho_{\text {int }}\left(C^{*}(\mathfrak{B})\right)
$$

satisfying

$$
\left.\mathcal{E}\left(\sum_{x \in G} \rho_{\text {int }}(a) \otimes \lambda_{x}\right)\right)=\rho_{\text {int }}(a) \quad\left(a \in C^{*}(\mathfrak{B})\right) .
$$

We have

$$
\mathcal{E}\left(a\left(1_{\mathcal{O}(X(\rho))} \otimes \lambda_{y}\right)\right)=\mathcal{E}\left(\left(1_{\mathcal{O}(X(\rho))} \otimes \lambda_{y}\right) a\right)
$$

for $a \in \rho_{\text {int }}\left(C^{*}(\mathfrak{B})\right) \otimes_{\min } C_{r}^{*}(G), x, y \in G$.

Remark 6.2. $C_{\text {Red }}^{*}(\mathfrak{B})=(\rho \otimes \lambda)_{\text {int }}\left(C^{*}(\mathfrak{B})\right)$ is a $C^{*}$ subalgebra of $\rho_{\text {int }}\left(C^{*}(\mathfrak{B})\right) \otimes_{\min } C_{r}^{*}(G)$, for we have

$\sum_{x \in G} \rho(f(x)) \otimes \lambda_{x} \in \rho_{\text {int }}\left(C^{*}(\mathfrak{B})\right) \otimes_{\min } C_{r}^{*}(G) \quad(f \in \mathfrak{L}(\mathfrak{B}))$.

Let $F: C_{\text {Red }}^{*}(\mathfrak{B}) \rightarrow C_{\text {Red }}^{*}(\mathfrak{B})$ be a completely positive map, then we can regard $F$ as a completely positive map on $(\rho \otimes \lambda)_{\text {int }}\left(C^{*}(\mathfrak{B})\right)$. Let $(V, r)$ be a representation of $F$ (see Definition 4.1), where $r: \rho_{\text {int }}\left(C^{*}(\mathfrak{B})\right) \rightarrow X(r)$, $V \in \mathcal{O}\left(\mathfrak{L}_{2}(G, X(\rho)), X(r)\right)$ (notice that $\rho$ weakly contains $r)$. Since it is easy to verify that $\mathcal{E}\left(F\left(\rho\left(a_{x}\right) \otimes \lambda_{x}\right)\left(1_{\mathcal{O}(X(\rho))} \otimes\right.\right.$ $\left.\left.\lambda_{x}\right)^{*}\right) \in B_{x}$ for all $x \in G$, we can define the multiplier $h_{F}: B \rightarrow B$ by

$$
h_{F}\left(a_{x}\right)=\mathcal{E}\left(F\left(\rho\left(a_{x}\right) \otimes \lambda_{x}\right)\left(1_{\mathcal{O}(X(\rho))} \otimes \lambda_{x}\right)^{*}\right) \quad\left(a_{x} \in B_{x}\right) .
$$

Proposition 6.2. $h_{F}$ is a completely positive Herz-Schur multiplier such that

$$
\left\|h_{F}\right\|_{\mathfrak{H} \cdot \mathfrak{S}} \leq\|F\|_{\mathrm{cb}}
$$

proof. Since $\left.\rho\right|_{B_{e}}$ is faithful, we identify $B_{e}$ with $\rho\left(B_{e}\right)$. We have

$$
\begin{aligned}
& \rho_{D}\left(N\left(h_{F}\right)((x, y ; a))\right) \\
= & \mathcal{E}\left(\left(\left(1_{\mathcal{O}(X(\rho))} \otimes \lambda_{x}\right) V^{*}\right) r(a)\left(V\left(1_{\mathcal{O}(X(\rho))} \otimes \lambda_{y}^{*}\right)\right)\right)
\end{aligned}
$$

for all $a \in B_{x y^{-1}}$. Since: (1) $\mathcal{E}$ is completely positive; (2) $r$ is weakly contained in $\rho$; (3) $\mathcal{E}$ is completely positive; (4) $x \mapsto V\left(1_{\mathcal{O}(X(\rho))} \otimes \lambda_{x}^{*}\right)$ is bounded, by Corollary $6.1, N\left(h_{F}\right)$ is a completely positive Schur $\rho$-multiplier. By Theorem 5.1, $h_{F}$ is completely positive Herz-Schur multiplier.

Furthermore, by 6.2 , we have

$$
\left\|h_{F}\right\|_{\mathfrak{H} \cdot \mathfrak{S}}=\left\|\left(h_{F}\right)_{e}\right\| \leq\|F\|_{\mathrm{cb}} .
$$

Theorem 6.1. The following are equivalent:

(i) $\mathfrak{B}$ is nuclear;

(ii) $C_{\text {Red }}^{*}(\mathfrak{B})$ is nuclear $C^{*}$-algebra.

If either of this hold, we have $C^{*}(\mathfrak{B})=C_{\text {Red }}^{*}(\mathfrak{B})$.

proof. By the aid of Proposition 6.2, we can prove the equivalence of $(i)$ and $(i i)$ by the argument of [9, Theorem 4.3]. If $(i i)$ holds, then by [4, Theorem 25.11] $C^{*}(\mathfrak{B})=$ $C_{\text {Red }}^{*}(\mathfrak{B})$.

Corollary 6.3. If $\mathfrak{B}$ is a Fell bundle over discrete group $G$ such that either $C^{*}(\mathfrak{B})$ or $C_{\text {Red }}^{*}(\mathfrak{B})$ is nuclear $C^{*}$-algebra, then for any subgroup $H \subset G, C^{*}\left(\mathfrak{B}_{H}\right)$ and $C_{\text {Red }}^{*}\left(\mathfrak{B}_{H}\right)$ is nuclear $C^{*}$-algebra. Furthermore, $\mathfrak{B}_{H}$ is amenable.

proof. Notice that if $C^{*}(\mathfrak{B})$ is nuclear, then $C_{\text {Red }}^{*}(\mathfrak{B})$ is nuclear because the quotient $C^{*}$-algebra of a nuclear $C^{*}$ algebra is nuclear.

If $C_{\text {Red }}^{*}(\mathfrak{B})$ is nuclear, then by Theorem 6.1 we have a 
net $\left\{\Psi_{i}\right\}_{i \in I}$ of Herz-Shur multipliers satisfying $(i)-($ iii $)$ of Defnition 6.1. It is easy to see that each $\left.\Psi_{i}\right|_{\mathfrak{B}_{H}}$ is completely positive Herz-Schur multiplier of $\mathfrak{B}_{H}$. Furthermore, since $\left\{\Psi_{i}\right\}_{i \in I}$ satisfies $(i)-($ iii $)$ of Definition 6.1, $\left\{\left.\Psi_{i}\right|_{\mathfrak{B}_{H}}\right\}$ satisfies $(i i)-(i i i)$ of Definition 6.1 , and by Corollary 6.2 $\left\{\left.\Psi_{i}\right|_{\mathfrak{B}_{H}}\right\}$ satisfies $(i)$ of Definition 6.1. By Theorem 6.1, we conclude that $C_{\mathrm{Red}}^{*}\left(\mathfrak{B}_{H}\right)$ is nuclear. By Theorem 6.1 again, $C_{\text {Red }}^{*}\left(\mathfrak{B}_{H}\right)=C^{*}\left(\mathfrak{B}_{H}\right)$, thus $\mathfrak{B}_{H}$ is amenable.

\section{Conclusion}

Our conclusion is that for a Fell bundle $\mathcal{B}$ over a discrete group $G$, the cross-sectional $C^{*}$-algebra $C^{*}(\mathcal{B})$ of $\mathcal{B}$ is nuclear if and only if $\mathcal{B}$ has Herz-Schur multipliers which we developed in this paper. Furthermore, we conclude that if $C^{*}(\mathcal{B})$ is a nuclear $C^{*}$-algebra, then for any subgroup $H \subset G$, $C^{*}\left(\mathcal{B}_{H}\right)$ is nuclear, where $\mathcal{B}_{H}$ is the restricted bundle of $\mathcal{B}$ on $H$.

\section{References}

[1] Marek Boz'ejko and Gero Fendler, Herz-schur multipliers and completely bounded multipliers of the fourier algebra of a locally compact group, Unione Matematica Italiana. Bollettino. A. Serie VI 3 (1984), no. 2 (1984).

[2] Nathanial P. Brown and Narutaka Ozawa, $C^{*}$-algebras and finite-dimensional approximations, Graduate Studies in Mathematics, vol. 88, American Mathematical Society, Providence, RI, 2008. MR2391387.

[3] Jean De Canniere and Uffe Haagerup, Multipliers of the fourier algebras of some simple lie groups and their discrete subgroups, American Journal of Mathematics 107 (1985).
[4] Ruy Exel, Partial dynamical systems, Fell bundles and applications, Mathematical Surveys and Monographs, vol. 224, American Mathematical Society, Providence, RI, 2017. MR3699795.

[5] Ruy Exel and Chi-Keung Ng, Approximation property of $C^{*}$-algebraic bundles, Math. Proc. Cambridge Philos. Soc. 132 (2002), no. 3, 509-522. MR1891686.

[6] J. M. G. Fell and R. S. Doran, Representations of *-algebras, locally compact groups, and Banach $C^{*}$ algebraic bundles. Vol. 1, Pure and Applied Mathematics, vol. 125, Academic Press, Inc., Boston, MA, 1988. Basic representation theory of groups and algebras. MR936628.

[7] J. M. G. Representations of ${ }^{*}$-algebras, locally compact groups, and Banach *-algebraic bundles. Vol. 2, Pure and Applied Mathematics, vol. 126, Academic Press, Inc., Boston, MA, 1988. Banach *-algebraic bundles, induced representations, and the generalized Mackey analysis. MR936629.

[8] A. Grothendieck, Rsum de la thorie mtrique des produits tensoriels topologiques, Boll. Soc. Mat. Sao-Paulo 8 (1956), 1-79.

[9] Andrew McKee, Adam Skalski, Ivan G. Todorov, and Lyudmila Turowska, Positive Herz-Schur multipliers and approximation properties of crossed products, Math. Proc. Cambridge Philos. Soc. 165 (2018), no. 3, 511532. MR3860401.

[10] Andrew McKee, Ivan Todorov, and Lyudmyla Turowska, Herz-Schur multipliers of dynamical systems (2016). Preprint. arXiv:1608.01092 [math.OA].

[11] Vern Paulsen, Completely bounded maps and operator algebras, Cambridge Studies in Ad-vanced Mathematics, vol. 78, Cambridge University Press, Cambridge, 2002. MR1976867. 